\title{
Influence of Magnetic Field, Viscous Dissipation and Thermophoresis on Darcy-Forcheimer Mixed Convection Flow in Fluid Saturated Porous Media
}

\author{
Adeyemi Isaiah Fagbade, Bidemi Olumide Falodun, Chika Uchechukwu Boneze \\ Department of Mathematical Sciences, Federal University of Technology, Akure, Nigeria \\ Email: yemi2favours@yahoo.co.uk, falodunbidemi2014@gmail.com, bonezechika@gmail.com
}

Received 10 February 2015; accepted 10 March 2015; published 12 March 2015

Copyright (C) 2015 by authors and Scientific Research Publishing Inc.

This work is licensed under the Creative Commons Attribution International License (CC BY).

http://creativecommons.org/licenses/by/4.0/

(c) (i) Open Access

\begin{abstract}
This paper presents an application of the spectral homotopy analysis method (SHAM) to solve a problem of darcy-forcheimer mixed convection flow in a porous medium in the presence of magnetic field, viscous dissipation and thermopherisis. A mathematical model governed the flow is analyzed in order to study the effects of chemical reaction, magnetic field, viscous dissipation and thermophoresis on mixed convection boundary layer flow of an incompressible, electrically conducting fluid past a heated vertical permeable flat plate embedded in a uniform porous medium. The similarity variable is used to transform the governing equations into a boundary valued problem of coupled ordinary differential equations which are then solved using spectral homotopy Analysis Method. The spatial domains are discretized using Chebyshev-Gauss-Lobatto points and numerical computations are carried out for the non-dimensional physical parameters. A parametric study of selected parameters is conducted and the results for the velocity, temperature and concentration are illustrated graphically and physical aspects of the problem are discussed.
\end{abstract}

\section{Keywords}

Darcy-Forcheimer, Porous Medium, Magnetic Effect, Chemical Reaction, Thermophoresis, Spectral Homotopy Analysis Method (SHAM)

\section{Introduction}

Mixed convective flow and heat transfer in saturated porous media is of practical interest in engineering

How to cite this paper: Fagbade, A.I., Falodun, B.O. and Boneze, C.U. (2015) Influence of Magnetic Field, Viscous Dissipation and Thermophoresis on Darcy-Forcheimer Mixed Convection Flow in Fluid Saturated Porous Media. American Journal of Computational Mathematics, 5, 18-40. http://dx.doi.org/10.4236/ajcm.2015.51002 
activities because of its wide applications as seen in porous insulation design, resin transfer modeling, nuclear waste disposal, etc. Over the years, the researchers in the field of fluid mechanics have intensified their research to unravel the importance of particles deposition technology due to its numerous engineering applications. Most of the research efforts as cited in [1]-[4] are concerned about free convection using Darcy's law, which states that the volume-averaged velocity is proportional to the pressure gradient. The Darcy model has shown to be valid under conditions of low velocities and small porosity. In many practical situations, the porous medium is bounded by an impermeable wall, high flow rates and non uniform porosity distribution in the near-wall region, thereby rendering Darcy's law inappropriate and inadequate. Hence, to model the real physical situations better, it is therefore necessary to include the non-Darcian effects in the analysis of convective transport in a porous medium.

The problem of Darcy Forchheimer mixed convection heat and mass transfer in fluid-saturated porous media in the presence of thermophoresis was studied by [5]. This phenomenon has been the subject of considerable study in the past. In optical fiber synthesis, thermophoresis has been identified as the principal mechanism of mass transfer as used in the technique of modified chemical vapor deposition (MCVD) [6]. Thermophoresis is a gaseous mixture of active precursors directed over a heated substrate where solid film deposits are located. In particular, the mathematical modeling of the deposition of silicon thin films using MCVD methods has been accelerated by the quality control measures enforced by the micro-electronics industry. Such topics involve a variety of complex fluid dynamical processes including thermophoretic transport of particles deposits, heterogenous/homogenous chemical reactions, homogenous particulate nucleation and coupled heat and energy transfer.

The migration of small particles in the direction of decreasing thermal gradient is called thermophoresis [7]. That is, a phenomenon which causes small particles to be driven away from a hot surface and towards a cold one. Small particles, such as dust, when suspended in a gas with a temperature gradient, experience a force in the direction of the temperature gradient. The velocity acquired by these particles is called thermophoretic velocity, and the force experienced by the suspended particles due to the temperature gradient is called thermophoretic force. The magnitudes of thermophoretic velocity and thermophoretic force are proportional to the temperature gradient and depend on thermal conductivity of aerosol particles, the carrier gas, the heat capacity of the gas, the thermophoretic coefficient and the Knudsen number. Due to thermophoresis, small micron sized particles are deposited on cold surfaces. In this process, the repulsion of particles from hot objects also takes place, and a particle-free layer is observed around hot bodies [8].

Thermophoresis is of practical importance in a variety of industrial and engineering applications including aerosol collection, nuclear reactor safety, corrosion of heat exchanger, and micro contamination control. The use of thermophoretic heaters has led to a reduction in chip failures. In the same vein, there is potential application of thermophoresis in removing radioactive aerosols from containment domes in the event of a nuclear reactor accident. [9] was one of the first to study the role of thermophoresis in the laminar flow of a viscous and incompressible fluid. He used the classical problem of flow over a flat plate to calculate deposition rates and showed that substantial changes in surface deposition can be obtained by increasing the difference between the surface and free stream temperatures. This was later followed by the effect of thermophoresis on particle deposition from a mixed convection flow onto a vertical plate by [10] and [11]. Also, [12] discussed the effect of surface mass transfer on mixed-convection flow past a heated vertical permeable flat plate with thermophoresis. [13] studied the effect of thermophoretic particle deposition in free convection boundary layer from a vertical flat plate embedded in a porous medium.

Mixed convection flow occurs frequently in nature. The temperature distribution varies from layer to layer and these types of flows have wide applications in industry, agriculture and oceanography. [14] investigated the numerical study on MHD mixed convective flow with dispersion and chemical reaction over a vertical plate in non-darcy porous medium. Transport processes in porous media play a significant roles in various applications such as in geothermal engineering, thermal insulation, energy conservations, chemical catalytic reactors and many others. In many transport processes in nature and in industrial applications, heat and mass transfer with variable viscosity is a consequence of buoyancy effects caused by diffusion of heat and chemical species. [15] investigated the thermophoresis and chemical reaction effects on non-darcy MHD mixed convective heat and mass transfer past a porous wedge in the presence of suction/injection. [16] considered coupled heat and mass transfer in Darcy-Forchheimer mixed convection from a vertical flat plate embedded in a fluid saturated porous medium under the effects of radiation and viscous dissipation. Research on combined heat and mass transfer 
find application in chemical processing equipment, formation and dispersion of fog, distribution of temperature and moisture over agricultural field as well as grooves of fruit trees, damage of crops due to freezing, food processing, cooling towers, chemically reactive vapor deposition boundary layers in optical materials processing etc. [17] investigated the chemical reaction and radiation effects of surface mass transfer on mixed convection flow past a heated vertical flat permeable plate with thermophoresis. [18] investigates the numerical study of thermophoresis on aerosol particle deposition from hiemenz flow through porous medium onto a stretching surface.

The influence of magnetic field on heat and mass transfer by natural convection from vertical surfaces in porous media with Soret and Dufour effects has been carried out by [19]. Analysis of MHD free convection flow along a vertical porous plate embedded in a porous medium with magnetic field and heat generation was investigated by [20]. Viscous dissipation changes the temperature distributions by playing a role like an energy source that leads to affect heat transfer rates. The merits on the effects of viscous dissipation depend on whether the plate is being cooled or heated. The work of [21] deals with the effect of the viscous dissipation term along with temperature dependent heat source or sink on momentum, heat and mass transfer visco-elastic fluid flow over an accelerating surface. [22] investigated the effects of chemical reaction and heat generation on MHD boundary layer flow of moving vertical plate with suction and dissipation. Effects of thermophoresis, practical deposition and chemical reaction on unsteady MHD mixed convective flow over a porous wedge in the presence of temperature dependent viscosity was investigated by [23]. [24] studied the thermophoresis particle deposition and variable viscosity effects on non-darcy free convection in fluid saturated porous media with uniform suction or injection. [25] investigated on the effectiveness of variable heat and mass fluxes on hydromagnetic free convection and mass transfer flow along an inclined permeable stretching surface with thermophoresis. Influence of viscous dissipation on free convection in a non-darcy porous medium saturated with Nano-fluid in the presence of magnetic field was investigated in [26]. [27] investigated the thermophoresis and viscous dissipation effects on Darcy-Forchheimer MHD mixed convection in a fluid saturated porous media.

The effects of magnetic field on a boundary layer control and on the performance of many systems using electrically conducting fluid such as MHD power generators, the cooling of nuclear reactors, plasma studies, purification of molten metals from non-metallic inclusion geothermal energy extraction etc.has been discussed extensively in some literatures. The laminar boundary layer on a moving continous flat surface in the presence of suction and magnetic field was studied by [28]. They observed the effect of magnetic field on boundary layer thickness and skin friction at the surface. Boundary layer flow along a flat plate is considered when a magnetic field acts perpendicular to the plate. Recently, [29] considered the effect of magnetic field on the boundary layer flow, heat and mass transfer of nanofluids over a stretching cylinder. [30] studied the effect of a uniform transverse magnetic field on the stagnation point flow over a stretching and vertical sheet. While, [31] studied the effect of magnetic field on mixed convection boundary layer flow over an exponentially shrinking vertical sheet with suction.

From the literature survey, the influence of magnetic field,viscous dissipation and thermophoresis on DarcyForchhemer mixed convection in fluid saturated porous media in the presence of chemical reaction have not been investigated so far. Hence,we result to study the influence of magnetic field and thermophoresis on darcyforchhemer mixed convection in fluid saturated porous media with viscous dissipation and chemical reaction is investigated using a novel SHAM approach. The transformed governing equations is solved using the spectral homotopy analysis method.

\section{Mathematical Formulation}

Consider a steady mixed convection boundary layer flow over a vertical flat plate of constant temperature $T_{w}$ and concentration $C_{w}$, which is embedded in a fluid-saturated porous medium of ambient temperature $T_{\infty}$ and concentration $C_{\infty}$, respectively. The x-coordinate is measured along the plate from its leading edge and the ycoordinate normal to it. Also, it is assumed that there exists a homogeneous first-order chemical reaction with a constant rate $K_{c}$ between the diffusing species and the fluid. It is assumed that the porous medium is homogeneous and present everywhere in local thermodynamic equilibrium. It is to be mentioned that the hole size of the porous plate is assumed to be constant. The fluid state properties and that of porous medium are assumed to be constant. Allowing both Brownian motion of particles and thermophoretic transport, the governing boundary layer equations are 


$$
\begin{gathered}
\frac{\partial u}{\partial x}+\frac{\partial v}{\partial y}=0, \\
\frac{\partial u}{\partial y}+\frac{C_{f} \sqrt{K_{1}}}{v} \frac{\partial u^{2}}{\partial y}= \pm g \frac{K_{1}}{v}\left(\beta_{t} \frac{\partial T}{\partial y}+\beta_{c} \frac{\partial C}{\partial y}\right), \\
u \frac{\partial T}{\partial x}+v \frac{\partial T}{\partial y}=\frac{\lambda_{g}}{\rho C_{p}} \frac{\partial^{2} T}{\partial y^{2}}+\frac{v}{C_{p}}\left(\frac{\partial u}{\partial y}\right)^{2}+\frac{\sigma^{*} \beta_{o}^{2}}{\rho c_{p}}(u)^{2}, \\
u \frac{\partial C}{\partial x}+v \frac{\partial C}{\partial y}=D_{m} \frac{\partial^{2} C}{\partial y^{2}}-\frac{\partial}{\partial y}\left(V_{T} C\right)-K_{c} C .
\end{gathered}
$$

The initial conditions at $y=0$ are given as

$$
u(x, 0)=U_{w}(0)=a x, \quad v(x, 0)=0, \quad T(x, 0)=T_{\omega}, \quad C(x, 0)=C_{\omega}
$$

The boundary conditions as $y \rightarrow \infty$ are:

$$
u(x, \infty) \rightarrow 0, \quad T(x, \infty) \rightarrow T_{\infty}, \quad C(x, \infty) \rightarrow C_{\infty} .
$$

In Equation (2), the plus sign corresponds to the case where the buoyancy force has a corresponding "aiding effect" on the flow and the minus sign correspond to "opposing flow" case. The term $V_{T}$ in Equation (4) following [32] can be defined as:

$$
V_{T}=-\frac{k^{*}}{T_{m}} \frac{\partial T}{\partial y}
$$

setting $C_{\infty}=0$ and following [33], the thermophoretic coefficient $k^{*}$ is defined as

$$
k^{*}=\frac{2 C_{s}\left(\frac{\lambda_{g}}{\lambda_{p}}+C_{1} K n\right) C_{1}}{\left(1+3 C_{m} K n\right)\left(1+2 \frac{\lambda_{g}}{\lambda_{p}}+2 C_{t} K n\right)}
$$

The following relations are now introduced for $u, v, \theta(\eta)$ and $\phi(\eta)$ as

$$
u=\frac{\partial \psi}{\partial y}, \quad v=-\frac{\partial \psi}{\partial x}, \quad \theta(\eta)=\frac{T-T_{\infty}}{T_{w}-T_{\infty}}, \quad \phi(\eta)=\frac{C-C_{\infty}}{C_{w}-C_{\infty}} .
$$

It is well known that boundary layer flows have a predominant flow direction and boundary layer thickness is small compared to a typical length in the main flow direction. Boundary layer thickness usually increases with increasing downstream distance, the basic equations are transformed in order to make the transformed boundary layer thickness a slowly varying function of $y$, with this objective, the governing partial differential Equations (1)-(4) are transformed by following dimensionless variables for the mixed convection regime:

$$
\begin{aligned}
& \eta=y \sqrt{\frac{a}{v}}, \quad u=\frac{\partial \psi}{\partial y}, \quad v=-\frac{\partial \psi}{\partial x}, \quad \psi=\sqrt{a v} x f(\eta), \\
& T=T_{\infty}+\left(T_{w}-T_{\infty}\right) \theta(\eta), \quad C=C_{\infty}+\left(C_{w}-C_{\infty}\right) \phi(\eta) .
\end{aligned}
$$

Here, $\psi(x, y)$ is the stream function. The first two mathematical relations of (10) satisfy continuity Equation (1). Upon substituting the above transformation (10) into the governing Equations (1)-(4) we obtain the following non-dimensional form

$$
\begin{gathered}
f^{\prime \prime}+2 \Lambda f^{\prime \prime}=R a_{x}\left(\theta^{\prime}+N \phi^{\prime}\right), \\
\theta^{\prime \prime}+\operatorname{Pr} \theta^{\prime} f+\operatorname{PrEc}\left(f^{\prime 2}+M f^{\prime 2}\right)=0, \\
\phi^{\prime \prime}+S_{c} \tau\left(\phi \theta^{\prime \prime}+\phi^{\prime} \theta^{\prime}\right)+S_{c} f \phi^{\prime}-\beta \phi=0,
\end{gathered}
$$


where the prime denote differentiation with respect to $\eta$ and the pertinent dimensionless parameters are

$$
\begin{aligned}
& M=\frac{\sigma \beta_{0}^{2}}{\rho a}, P_{r}=\frac{\rho C_{p}}{\lambda_{g}}, S_{c}=\frac{v}{D_{m}}, \beta=\frac{K_{c} S_{c}}{a}, E_{c}=\frac{U_{w}^{2}}{C_{p}\left(T_{w}-T_{\infty}\right)}, \tau=-\frac{K\left(T_{w}-T_{\infty}\right)}{T_{m}}, \\
& \Lambda=\frac{a x C_{f} \sqrt{K_{1}}}{v}, R a_{x}=\frac{g K_{1} \beta_{t}\left(T_{w}-T_{\infty}\right)}{a x v}, N=\frac{\beta_{c}\left(C_{w}-C_{\infty}\right)}{\beta_{t}\left(T_{w}-T_{\infty}\right)} .
\end{aligned}
$$

Also using the above stated similarity transformations, the transformed boundary conditions are:

$$
\begin{aligned}
& f(0)=0, \quad \theta(0)=1, \quad \phi(0)=1, \\
& f^{\prime}(\infty)=0, \quad \theta(\infty)=0, \quad \phi(\infty)=0,
\end{aligned}
$$

The physical quantities of interest in this problem are Nusselt number and Sherwood number which are defined respectively as

Nusselt number:

$$
N u=-\sqrt{R e_{X}} \theta^{\prime}(0)
$$

Sherwood number:

$$
S h=-\sqrt{R e_{x}} \phi^{\prime}(0)
$$

\section{Approximate Method}

In this paper, the numerical version of the homotopy analysis method called SHAM is used to solve a set of ordinary differential equations (ODEs) that models the problem of darcy-forcheimer mixed convection flow over a vertical plate embedded in a saturated porous medium in the presence of Magnetic field and chemical reaction. SHAM is a numerical version of the homotopy analysis method (HAM) that has been widely applied to solve a wide variety of nonlinear ordinary and partial differential equation with applications in applied mathematics, physics, nonlinear mechanics, finance and engineering. A detailed systematic description of the HAM and its applications can be found in two books (and a huge list of references cited therein) ([34] [35]) by S. J. Liao who is credited with developing the method.

In essence, the HAM works by transforming a nonlinear ODE or PDE into an infinite number of linear ODEs which should be solvable analytical. The HAM solutions are required to conform to a predefined rule of solution expression. The SHAM was introduced in ([36] [37]) and it uses the principles of the traditional HAM and combines them with the Chebyshev spectral collocation method which is used in the solution of the transformed sequence of ordinary differential equations. One of the main features of the SHAM is the removal of the requirement to conform to a particular rule of solution expression. The SHAM also requires that the linear operator to be used in the development of the algorithm be selected as the entire linear part of the governing differential equation. This often leads to complicated sequence of linear ordinary differential equations which can only be solved numerically. Hence, the use of the Chebyshev spectral collocation method. Spectral methods are now becoming the preferred tools for solving ordinary and partial differential equations because of their elegance and high accuracy in resolving problems with smooth functions ([38] [39]).

In applying the spectral-homotopy analysis method, it is convenient to first transform the domain of the problem from $[0,1]$ to $[-1,1]$ and make the governing boundary conditions homogeneous by using the transformations

$$
\begin{gathered}
\xi=\frac{2 \eta}{L}-1, \quad \xi \in[-1,1], \quad f(\eta)=f(\xi)+f_{0}(\eta), \\
\theta(\eta)=\theta(\xi)+\theta_{0}(\eta), \quad \phi(\eta)=\phi(\xi)+\phi_{0}(\eta) \\
f_{0}(\eta)=+1-\mathrm{e}^{-\eta}, \quad \theta_{0}(\eta)=\mathrm{e}^{-\eta}, \quad \phi_{0}(\eta)=\mathrm{e}^{-\eta} .
\end{gathered}
$$

Substituting (15)-(16) in the governing equation and boundary conditions (11)-(14) gives

$$
f^{\prime \prime}+2 \Lambda f^{\prime \prime}+a_{1} \Lambda f^{\prime}+a_{2} \Lambda f^{\prime \prime}-R a_{x} \theta^{\prime}-R a_{x} N \phi^{\prime}=H_{1}(\eta)
$$




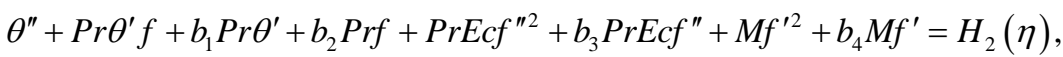

$$
\begin{aligned}
& \phi^{\prime \prime}-S_{c} \tau \phi \theta^{\prime \prime}-c_{1} S_{c} \tau \phi-c_{2} S_{c} \tau \theta^{\prime \prime}-S_{c} \tau \phi^{\prime} \theta^{\prime}-c_{3} S_{c} \tau \phi^{\prime} \\
& -c_{4} S_{c} \tau \theta^{\prime}+S_{c} f \phi^{\prime}+c_{4} S_{c} f+c_{5} S_{c} \phi^{\prime}-\beta \phi=H_{3}(\eta) \text {. }
\end{aligned}
$$

Subject to

$$
f(-1)=f^{\prime}(1)=0, \quad \theta(-1)=\theta(1)=0, \quad \phi(-1)=\phi(1)=0
$$

where the primes denote differentiation with respect to $\xi$ and

$$
\begin{aligned}
& a_{1}=2 f_{0}^{\prime \prime}(\eta), \quad a_{2}=2 f_{0}^{\prime}(\eta), \quad a_{3}=0, \quad a_{4}=0, \quad a_{5}=0, \\
& b_{1}=f_{0}(\eta), \quad b_{2}=\theta_{0}^{\prime}(\eta) . \quad b_{3}=2 f_{0}^{\prime \prime}(\eta), \quad b_{4}=2 f_{0}^{\prime}(\eta), \quad b_{5}=0 \\
& c_{1}=\theta_{0}^{\prime \prime}(\eta), \quad c_{2}=\phi_{0}(\eta), \quad c_{3}=\theta_{0}^{\prime}(\eta), \quad c_{4}=\phi_{0}^{\prime}(\eta), \quad c_{5}=f_{0}(\eta) .
\end{aligned}
$$

And

$$
\begin{aligned}
& H_{1}(\eta)=R a_{x} \theta_{0}^{\prime}+R a_{x} N \phi_{0}^{\prime}-2 \Lambda f_{0}^{\prime} f_{0}^{\prime \prime}-f_{0}^{\prime \prime}, \\
& H_{2}(\eta)=-\theta_{0}^{\prime \prime}-\operatorname{Pr} \theta_{0}^{\prime} f_{0}-\operatorname{Pr} E c f_{0}^{\prime \prime 2}-M f_{0}^{\prime 2} \text {, } \\
& H_{3}(\eta)=\beta \phi_{0}-S_{c} f_{0} \phi_{0}^{\prime}-S_{c} \tau \phi_{0}^{\prime} \theta_{0}^{\prime}-S_{c} \tau \phi_{0} \theta_{0}^{\prime \prime}-\phi_{0}^{\prime \prime} .
\end{aligned}
$$

The initial approximation is taken to be the solution of the nonhomogeneous linear part of the governing Equations (17)-(19) given by

$$
\begin{gathered}
f_{l}^{\prime \prime}+a_{1} \Lambda f_{l}^{\prime \prime}+a_{2} \Lambda f_{l}^{\prime \prime}-R a_{x} \theta_{l}^{\prime}-R a_{x} N \phi_{l}^{\prime}=H_{1}(\eta), \\
\theta_{l}^{\prime \prime}+b_{1} \operatorname{Pr} \theta_{l}^{\prime}+b_{2} \operatorname{Pr} f_{l}+b_{3} \operatorname{Pr} E c f_{l}^{\prime \prime}+b_{4} M f_{l}^{\prime}=H_{2}(\eta), \\
\phi_{l}^{\prime \prime}-c_{1} S_{c} \tau \phi_{l}-c_{2} S_{c} \tau \theta_{l}^{\prime \prime}-c_{3} S_{c} \tau \phi_{l}^{\prime}-c_{4} S_{c} \tau \theta_{l}^{\prime}+c_{4} S_{c} f_{l}+c_{5} S_{c} \phi_{l}^{\prime}-\beta \phi_{l}=H_{3}(\eta) .
\end{gathered}
$$

subject to

$$
f_{l}(-1)=f_{l}^{\prime}(1)=0, \quad \theta_{l}(-1)=\theta_{l}(1)=0, \quad \phi_{l}(-1)=\phi_{l}(1)=0
$$

We use the Chebyshev pseudospectral method to solve (25)-(28). The unknown function $f_{l}(\xi), \theta_{l}(\xi)$ and $\phi_{l}(\xi)$ are approximated as a truncated series of Chebyshev polynomials of the form:

$$
\begin{array}{ll}
f_{l}(\xi) \approx f_{l}^{N}\left(\xi_{j}\right)+\sum_{k=0}^{N} \hat{f}_{k} T_{k}\left(\xi_{j}\right) & j=0,1, \cdots, N, \\
\theta_{l}(\xi) \approx \theta_{l}^{N}\left(\xi_{j}\right)+\sum_{k=0}^{N} \hat{\theta}_{k} T_{k}\left(\xi_{j}\right) & j=0,1, \cdots, N, \\
\phi_{l}(\xi) \approx \phi_{l}^{N}\left(\xi_{j}\right)+\sum_{k=0}^{N} \hat{\phi}_{k} T_{k}\left(\xi_{j}\right) & j=0,1, \cdots, N
\end{array}
$$

where $T_{k}$ is the kth Chebyshev polynomial, $\hat{f}_{k}, \hat{\theta}_{k}$ and $\hat{\phi}_{k}$ are coefficients and $\xi_{0}, \xi_{1}, \cdots, \xi_{N}$ are GaussLobatto collocation points (see [28]) defined by

$$
\xi_{j}=\cos \left(\frac{\pi j}{N}\right), \quad j=0,1, \cdots, N .
$$

Derivatives of the functions $f_{l}(\xi), \theta_{l}(\xi)$ and $\phi_{l}(\xi)$ at the collocation points are represented as

$$
\frac{\mathrm{d}^{r} f_{l}}{\mathrm{~d} \xi^{r}}=\sum_{k=0}^{N} \boldsymbol{D}_{k j} f_{l}\left(\xi_{j}\right), \quad \frac{\mathrm{d}^{r} \theta_{l}}{\mathrm{~d} \xi^{r}}=\sum_{k=0}^{N} \boldsymbol{D}_{k j} \theta_{l}\left(\xi_{j}\right), \quad \frac{\mathrm{d}^{r} \phi_{l}}{\mathrm{~d} \xi^{r}}=\sum_{k=0}^{N} \boldsymbol{D}_{k j} \phi_{l}\left(\xi_{j}\right)
$$

where $r$ is the order of differentiation and $\boldsymbol{D}$ is the Chebyshev spectral differentiation matrix. Substituting the 
above expressions in (25)-(28) yields

$$
A F_{l}=\boldsymbol{G}
$$

Subject to

$$
\begin{gathered}
f_{l}\left(\xi_{N}\right)=0, \quad \sum_{k=0}^{N} \boldsymbol{D}_{0 k} f_{l}\left(\xi_{k}\right)=0, \quad \theta_{l}\left(\xi_{N}\right)=0, \quad \theta_{l}\left(\xi_{0}\right)=0 \\
\phi_{l}\left(\xi_{N}\right)=0, \quad \phi_{l}\left(\xi_{0}\right)=0
\end{gathered}
$$

where

$$
\boldsymbol{A}=\left(\begin{array}{lll}
A_{1,1} & A_{1,2} & A_{1,3} \\
A_{2,1} & A_{2,2} & A_{2,3} \\
A_{3,1} & A_{3,2} & A_{3,3}
\end{array}\right)
$$

And

$$
\begin{gathered}
A_{1,1}=\boldsymbol{D}^{2}+a_{1} \Lambda \boldsymbol{D}+a_{2} \Lambda \boldsymbol{D}^{2} \\
A_{1,2}=-R a_{x} \boldsymbol{D} \\
A_{1,3}=-R a_{x} N \boldsymbol{D} \\
A_{2,1}=b_{3} \operatorname{Pr} E \boldsymbol{c} \boldsymbol{D}^{2}+b_{4} M \boldsymbol{D}+b_{2} P r \\
A_{2,2}=\boldsymbol{D}^{2}+b_{1} \operatorname{Pr} \boldsymbol{D} \\
A_{2,3}=0 \\
A_{3,1}=c_{4} S_{c} \\
A_{3,2}=c_{2} S_{c} \tau \boldsymbol{D}^{2}+c_{4} S_{c} \tau \boldsymbol{D} \\
\left.\boldsymbol{F}_{l}=\left[f_{l}\left(\xi_{0}\right), f_{l}\left(\xi_{1}\right), \cdots, \boldsymbol{D}_{l}^{2}+c_{3} S_{c} \tau \boldsymbol{D}+c_{5} S_{c}\right), \theta_{l}\left(\xi_{0}\right), \theta_{l}\left(\xi_{1}\right), \cdots, \theta_{l}\left(\xi_{N}\right), \phi_{l}\left(\xi_{0}\right), \phi_{l}\left(\xi_{1}\right), \cdots, \phi_{l}\left(\xi_{N}\right)\right]^{\mathrm{T}} \\
\boldsymbol{G}=\left[H_{1}\left(\eta_{0}\right), H_{1}\left(\eta_{1}\right), \cdots, H_{1}\left(\eta_{N}\right), H_{2}\left(\eta_{0}\right), H_{2}\left(\eta_{1}\right), \cdots, H_{2}\left(\eta_{N}\right), H_{3}\left(\eta_{0}\right), H_{3}\left(\eta_{1}\right), \cdots, H_{3}\left(\eta_{N}\right)\right] \\
a_{i}=\operatorname{diag}\left(\left[a_{i}\left(\eta_{0}\right), a_{i}\left(\eta_{1}\right), a_{i}\left(\eta_{1}\right), \cdots, a_{i}\left(\eta_{N-1}\right), a_{i}\left(\eta_{N}\right)\right]\right) \\
b_{i}=\operatorname{diag}\left(\left[b_{i}\left(\eta_{0}\right), b_{i}\left(\eta_{1}\right), b_{i}\left(\eta_{1}\right), \cdots, b_{i}\left(\eta_{N-1}\right), b_{i}\left(\eta_{N}\right)\right]\right) \\
c_{i}=\operatorname{diag}\left(\left[c_{i}\left(\eta_{0}\right), c_{i}\left(\eta_{1}\right), c_{i}\left(\eta_{1}\right), \ldots, c_{i}\left(\eta_{N-1}\right), c_{i}\left(\eta_{N}\right)\right]\right)
\end{gathered}
$$

$i=1,2,3,4,5$. In the above definitions, the superscript $T$ denotes transpose, diag is a diagonal matrix and $I$ is an identity matrix of size $(N+1) \times(N+1)$ To implement the boundary conditions (33), we delete the first and the last rows and columns of $A$ and delete the first and last rows of $f_{l}(\xi), \theta_{l}(\xi), \phi_{l}(\xi)$ and $\boldsymbol{G}$. The boundary conditions (33) are imposed on the resulting first and last rows of the modified matrix $\boldsymbol{A}$ and setting the resulting first and last rows of the modified matrix $\boldsymbol{G}$ to be zero. The values of $f_{l}\left(\xi_{0}\right), f_{l}\left(\xi_{1}\right), \cdots, f_{l}\left(\xi_{N}\right), \theta_{l}\left(\xi_{0}\right), \theta_{l}\left(\xi_{1}\right), \cdots, \theta_{l}\left(\xi_{N}\right), \phi_{l}\left(\xi_{0}\right), \phi_{l}\left(\xi_{1}\right), \cdots, \phi_{l}\left(\xi_{N}\right)$ are then determined from

$$
\boldsymbol{F}_{l}=\boldsymbol{A}^{-1} \boldsymbol{G}
$$

To find the SHAM solutions of (17)-(19), we begin by defining the following linear operator:

$$
L_{f}[\bar{f}(\eta ; q), \bar{\theta}(\eta ; q), \bar{\phi}(\eta ; q)]=f^{\prime \prime}+a_{1} \Lambda f^{\prime}+a_{2} \Lambda f^{\prime \prime}-R a_{x} \theta^{\prime}-R a_{x} N \phi^{\prime},
$$




$$
\begin{gathered}
L_{\theta}[\bar{f}(\eta ; q), \bar{\theta}(\eta ; q), \bar{\phi}(\eta ; q)]=\theta^{\prime \prime}+b_{1} \operatorname{Pr} \theta^{\prime}+b_{2} \operatorname{Prf}+b_{3} \operatorname{PrEcf} f^{\prime \prime}+b_{4} M f^{\prime}, \\
L_{\phi}[\bar{f}(\eta ; q), \bar{\theta}(\eta ; q), \bar{\phi}(\eta ; q)]=\phi^{\prime \prime}-c_{1} S_{c} \tau \phi-c_{2} S_{c} \tau \theta^{\prime \prime}-c_{3} S_{c} \tau \phi^{\prime}-c_{4} S_{c} \tau \theta^{\prime}+c_{4} S_{c} f+c_{5} S_{c} \phi^{\prime}-\beta \phi .
\end{gathered}
$$

$q \in[0,1]$ is an embedding parameter and $\bar{f}(\eta, q), \bar{\theta}(\eta, q)$ and $\bar{\phi}(\eta, q)$ are the unknown functions. The zeroth order deformation is given as

$$
\begin{aligned}
& (1-q) L_{\bar{f}}\left[\bar{f}(\eta ; q)-\bar{f}_{0}(\eta)\right]=q \hbar_{\bar{f}} H_{\bar{f}}(\eta) N_{\hbar_{\bar{f}}}[\bar{f}(\eta ; q), \bar{\theta}(\eta ; q), \bar{\phi}(\eta ; q)], \\
& (1-q) L_{\bar{\theta}}\left[\bar{\theta}(\eta ; q)-\bar{\theta}_{0}(\eta)\right]=q \hbar_{\bar{\theta}} H_{\bar{\theta}}(\eta) N_{\hbar_{\bar{\theta}}}[\bar{f}(\eta ; q), \bar{\theta}(\eta ; q), \bar{\phi}(\eta ; q)], \\
& (1-q) L_{\bar{\phi}}\left[\bar{\phi}(\eta ; q)-\bar{\phi}_{0}(\eta)\right]=q \hbar_{\bar{\phi}} H_{\bar{\phi}}(\eta) N_{\hbar_{\bar{\phi}}}[\bar{f}(\eta ; q), \bar{\theta}(\eta ; q), \bar{\phi}(\eta ; q)] .
\end{aligned}
$$

where $\hbar_{\bar{f}}, \hbar_{\bar{\theta}}$ and $\hbar_{\bar{\phi}}$ are the nonzero convergence controlling auxiliary parameters and $N_{\hbar_{\bar{f}}}, N_{\hbar_{\bar{\theta}}}$ and $N_{\hbar_{\bar{\phi}}}$ are nonlinear operators given by

$$
\begin{aligned}
N_{\hbar_{\bar{f}}}[\bar{f}(\eta ; q), \bar{\theta}(\eta ; q), \bar{\phi}(\eta ; q)]= & \bar{f}^{\prime \prime}+2 \Lambda \bar{f}^{\prime} \bar{f}^{\prime \prime}+a_{1} \Lambda \bar{f}^{\prime}+a_{2} \Lambda \bar{f}^{\prime \prime}-R a_{x} \bar{\theta}^{\prime}-R a_{x} N \bar{\phi}^{\prime}, \\
N_{\hbar_{\bar{\theta}}}[\bar{f}(\eta ; q), \bar{\theta}(\eta ; q), \bar{\phi}(\eta ; q)]= & \bar{\theta}^{\prime \prime}+\operatorname{Pr} \bar{\theta}^{\prime} \bar{f}+b_{1} \operatorname{Pr} \bar{\theta}^{\prime}+b_{2} \operatorname{Pr} \bar{f}+\operatorname{PrEc} \bar{f}^{\prime \prime 2} \\
& +b_{3} \operatorname{Pr} E c \bar{f}^{\prime \prime}+M \bar{f}^{\prime 2}+b_{4} M \overline{f^{\prime}}, \\
N_{\hbar_{\bar{\phi}}}[\bar{f}(\eta ; q), \bar{\theta}(\eta ; q), \bar{\phi}(\eta ; q)]= & \bar{\phi}^{\prime \prime}-S_{c} \tau \overline{\phi \theta^{\prime \prime}}-c_{1} S_{c} \tau \bar{\phi}-c_{2} S_{c} \tau \bar{\theta}^{\prime \prime}-S_{c} \tau \bar{\phi}^{\prime} \bar{\theta}^{\prime}-c_{3} S_{c} \tau \bar{\phi}^{\prime} \\
& -c_{4} S_{c} \tau \bar{\theta}^{\prime}+S_{c} \bar{f} \bar{\phi}^{\prime}+c_{4} S_{c} \bar{f}+c_{5} S S_{c} \bar{\phi}^{\prime}-\beta \bar{\phi} .
\end{aligned}
$$

Differentiating (38)-(40) $m$ times with respect to $\mathrm{q}$ and then setting $q=0$ and finally dividing the resulting equations by $m$ ! yields the mth order deformation equations:

$$
\begin{aligned}
& L_{\bar{f}}\left[\bar{f}(\xi)-\chi_{m} \bar{f}_{m-1}(\xi)\right]=\hbar_{\bar{f}} H_{\bar{f}}(\xi) R_{m}^{\bar{f}}(\xi), \\
& L_{\bar{\theta}}\left[\bar{\theta}(\xi)-\chi_{m} \bar{\theta}_{m-1}(\xi)\right]=\hbar_{\bar{\theta}} H_{\bar{\theta}}(\xi) R_{m}^{\bar{\theta}}(\xi), \\
& L_{\bar{\phi}}\left[\bar{\phi}(\xi)-\chi_{m} \bar{\phi}_{m-1}(\xi)\right]=\hbar_{\bar{\phi}} H_{\bar{\phi}}(\xi) R_{m}^{\bar{\phi}}(\xi) .
\end{aligned}
$$

Subject to

$$
\begin{aligned}
& \bar{f}_{m}(-1)=0, \quad \bar{\theta}_{m}(-1)=0, \quad \bar{\phi}_{m}(-1)=1, \\
& \bar{f}_{m}^{\prime}(1)=0, \quad \bar{\theta}_{m}(1)=0, \quad \bar{\phi}_{m}(1)=0 .
\end{aligned}
$$

where

$$
\begin{aligned}
R_{m}^{\bar{f}}(\xi)=\bar{f}_{m-1}^{\prime \prime} & +a_{1} \Lambda \bar{f}_{m-1}^{\prime}+a_{2} \Lambda \bar{f}_{m-1}^{\prime \prime}-R a_{x} \bar{\theta}_{m-1}^{\prime}-R a_{x} N \bar{\phi}_{m-1}^{\prime}+2 \Lambda \sum_{n=1}^{m-1} \bar{f}_{n}^{\prime} \bar{f}_{m-1-n}^{\prime \prime}-H_{1}(\eta)\left(1-\chi_{m}\right), \\
R_{m}^{\bar{\theta}}(\xi)= & \bar{\theta}_{m-1}^{\prime \prime}+b_{1} \operatorname{Pr} \bar{\theta}_{m-1}^{\prime}+b_{2} \operatorname{Pr} \bar{f}_{m-1}+b_{3} \operatorname{PrEc} \bar{f}_{m-1}^{\prime \prime}+b_{4} M \bar{f}_{m-1}^{\prime} \\
& +\sum_{n=1}^{m-1}\left[\operatorname{Pr} \bar{\theta}_{n}^{\prime} \bar{f}_{m-1-n}+\operatorname{PrEc} \bar{f}_{n}^{\prime \prime} \bar{f}_{m-1 n}^{\prime \prime}+M \bar{f}_{n}^{\prime} \bar{f}_{m-1-n}^{\prime}\right]-H_{2}(\eta)\left(1-\chi_{m}\right) \\
R_{m}^{\bar{\phi}}(\xi)= & \bar{\phi}_{m-1}^{\prime \prime}+c_{1} S_{c} \tau \bar{\phi}_{m-1}+c_{2} S_{c} \tau \bar{\theta}_{m-1}^{\prime \prime}+c_{3} S_{c} \tau \bar{\phi}_{m-1}^{\prime}+c_{4} S_{c} \bar{f}_{m-1}+c_{5} S_{c} \bar{\phi}_{m-1}^{\prime}-\beta \bar{\phi}_{m-1} \\
& +c_{4} S_{c} \tau \bar{\theta}_{m-1}^{\prime}+\sum_{n=1}^{m-1}\left[S_{c} \tau \bar{\phi}_{n} \bar{\theta}_{m-1-n}^{\prime \prime}+S_{c} \tau \bar{\theta}_{n}^{\prime} \bar{\phi}_{m-1 n}^{\prime}\right]-H_{3}(\eta)\left(1-\chi_{m}\right) .
\end{aligned}
$$

And

$$
\chi_{m}= \begin{cases}0 & \text { when } m \leq 0 \\ 1 & \text { when } m>1\end{cases}
$$


Applying the Chebyshev pseudospectral transformation on (44)-(46) gives

$$
\begin{aligned}
\boldsymbol{A} \overline{\boldsymbol{f}}_{m} & =\left(\chi_{m}+\hbar_{\bar{f}}\right) \boldsymbol{A} \overline{\boldsymbol{f}}_{m-1}-\hbar_{\bar{f}}\left(1-\chi_{m}\right) \boldsymbol{G}+\hbar_{\bar{f}} P_{m-1}^{\overline{\bar{f}}} \\
\boldsymbol{A} \bar{\theta}_{m} & =\left(\chi_{m}+\hbar_{\bar{\theta}}\right) \boldsymbol{A} \bar{\theta}_{m-1}-\hbar_{\bar{\theta}}\left(1-\chi_{m}\right) \boldsymbol{G}+\hbar_{\bar{\theta}} P_{m-1}^{\bar{\theta}} \\
\boldsymbol{A} \bar{\phi}_{m} & =\left(\chi_{m}+\hbar_{\bar{\phi}}\right) \boldsymbol{A} \bar{\phi}_{m-1}-\hbar_{\bar{\phi}}\left(1-\chi_{m}\right) \boldsymbol{G}+\hbar_{\bar{\phi}} P_{m-1}^{\bar{\phi}}
\end{aligned}
$$

Subject to

$$
\begin{gathered}
\bar{f}_{m}\left(\xi_{N}\right)=0, \quad \sum_{k=0}^{N} \boldsymbol{D}_{0 k} \bar{f}_{m}\left(\xi_{k}\right)=0, \bar{\theta}_{m}\left(\xi_{N}\right)=0, \\
\bar{\theta}_{m}\left(\xi_{0}\right)=0, \quad \bar{\phi}_{m}\left(\xi_{N}\right)=0, \quad \bar{\phi}_{m}\left(\xi_{0}\right)=0
\end{gathered}
$$

where $\boldsymbol{A}$ and $\boldsymbol{G}$, are as defined above and

$$
\begin{gathered}
\overline{\boldsymbol{F}}_{m}=\left[\bar{f}_{m}\left(\xi_{0}\right), \bar{f}_{m}\left(\xi_{1}\right), \cdots, \bar{f}_{m}\left(\xi_{N}\right), \bar{\theta}_{m}\left(\xi_{0}\right), \bar{\theta}_{m}\left(\xi_{1}\right), \cdots, \bar{\theta}_{m}\left(\xi_{N}\right), \bar{\phi}_{m}\left(\xi_{0}\right), \bar{\phi}_{m}\left(\xi_{1}\right), \cdots, \bar{\phi}_{m}\left(\xi_{N}\right)\right]^{\mathrm{T}} \\
P_{m-1}^{\bar{f}}=\sum_{n=0}^{m-1}\left[2 \Lambda\left(\boldsymbol{D} \bar{f}_{n}\right)\left(\boldsymbol{D}^{2} \bar{f}_{m-1-n}\right)\right] \\
P_{m-1}^{\bar{\theta}}=\sum_{n=0}^{m-1}\left[\operatorname{Pr}\left(\boldsymbol{D} \bar{\theta}_{n}\right)\left(\bar{f}_{m-1-n}\right)+\operatorname{PrEc}\left(\boldsymbol{D}^{2} \bar{f}_{n}\right)\left(\boldsymbol{D}^{2} \bar{f}_{m-1-n}\right)+M\left(\boldsymbol{D} \bar{f}_{n}\right)\left(\boldsymbol{D} \overline{\boldsymbol{f}}_{m-1-n}\right)\right] \\
P_{m-1}^{\bar{\phi}}=\sum_{n=0}^{m-1}\left[S_{c} \tau\left(\bar{\phi}_{n}\right)\left(\boldsymbol{D}^{2} \bar{\phi}_{m-1-n}\right)+S_{c} \tau\left(\boldsymbol{D} \bar{\theta}_{n}\right)\left(\boldsymbol{D} \bar{\phi}_{m-1-n}\right)+S_{c}\left(\bar{f}_{n}\right)\left(\boldsymbol{D} \bar{\phi}_{m-1-n}\right)\right]
\end{gathered}
$$

To implement the boundary conditions above we delete the first and last rows of $P_{m-1}^{\bar{f}}, P_{m-1}^{\bar{\theta}}, P_{m-1}^{\bar{\phi}}$ and $\boldsymbol{G}$ and delete the first and last rows and first and last columns of $\boldsymbol{A}$ in 52, 53 and 54. The boundary conditions (54a) are imposed on the first and last row of the modified $\boldsymbol{A}$ matrix on the left side of the equal sign in 52, 53 and 54. The first and last rows of the modified $\boldsymbol{A}$ matrix on the right of the equal sign in 52, 53 and 54 are the set to be zero. This results in the following recursive formulas

$$
\begin{aligned}
& \bar{f}_{m}=\left(\chi_{m}+\hbar_{\bar{f}}\right) \boldsymbol{A}^{-1} \overline{\boldsymbol{A}}_{m-1}+\hbar_{\bar{f}} \boldsymbol{A}^{-1}\left[P_{m-1}^{\bar{f}}-\left(1-\chi_{m}\right) \boldsymbol{G}\right], \\
& \bar{\theta}_{m}=\left(\chi_{m}+\hbar_{\bar{\theta}}\right) \boldsymbol{A}^{-1} \overline{\boldsymbol{A}} \bar{\theta}_{m-1}+\hbar_{\bar{\theta}} \boldsymbol{A}^{-1}\left[P_{m-1}^{\bar{\theta}}-\left(1-\chi_{m}\right) \boldsymbol{G}\right], \\
& \bar{\phi}_{m}=\left(\chi_{m}+\hbar_{\bar{\phi}}\right) \boldsymbol{A}^{-1} \overline{\boldsymbol{A}} \bar{\phi}_{m-1}+\hbar_{\bar{\phi}} \boldsymbol{A}^{-1}\left[P_{m-1}^{\bar{\phi}}-\left(1-\chi_{m}\right) \boldsymbol{G}\right],
\end{aligned}
$$

Thus, starting from the initial approximation,obtain from (34), higher-order approximations $\bar{f}_{m}(\xi), \bar{\theta}_{m}(\xi)$, $\bar{\phi}_{m}(\xi)$ for $m \geq 1$ can be obtained through the recursive formula (58)-(60).

\section{Results and Discussion}

For the problem under investigation, numerical computations are carried out for different flow parameters such as inertia parameter $\Lambda$, thermal rayleigh number $R a_{x}$, buoyancy ratio $N$, Schmidt number $S c$, thermophoretic parameter $\tau$, Eckert number $E c$, Chemical reaction parameter $\beta$, Magnetic parameter $M$ and the results are reported in graphs and tables. This is done to illustrate special features of the numerical solutions. In this computation except otherwise stated, we used prandtl number $\operatorname{Pr}=0.73$, Schmidt number Sc $=0.62, \Lambda=1, N=2$, $h_{f}=h_{\theta}=h_{\phi}=-0.5, E c=0.01, M=0.5, R a_{x}=0.8, \beta=0.5$ and $\tau=0.01$. In addition the boundary condition $\eta \rightarrow \infty$ is approximated by $\eta_{\max }=20$; which is sufficiently large for the velocity to approach the relevant stream velocity.

Figures 1-8 illustrate the effects of the various pertinent parameters on the dimensionless velocity $f^{\prime}(\eta)$, temperature $\theta(\eta)$ and concentration $\phi(\eta)$. Figures 1(a)-(c) show the effect of the mixed convection 


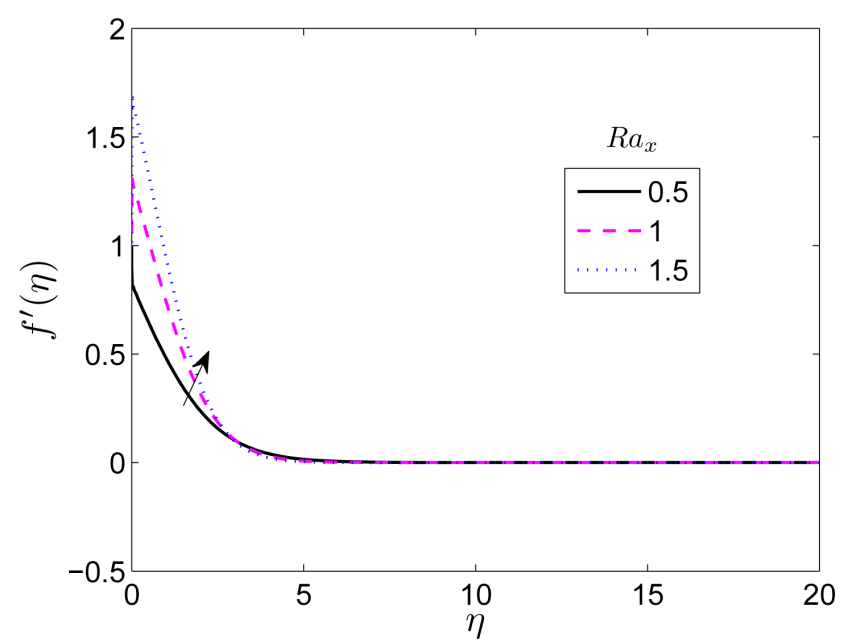

(a)

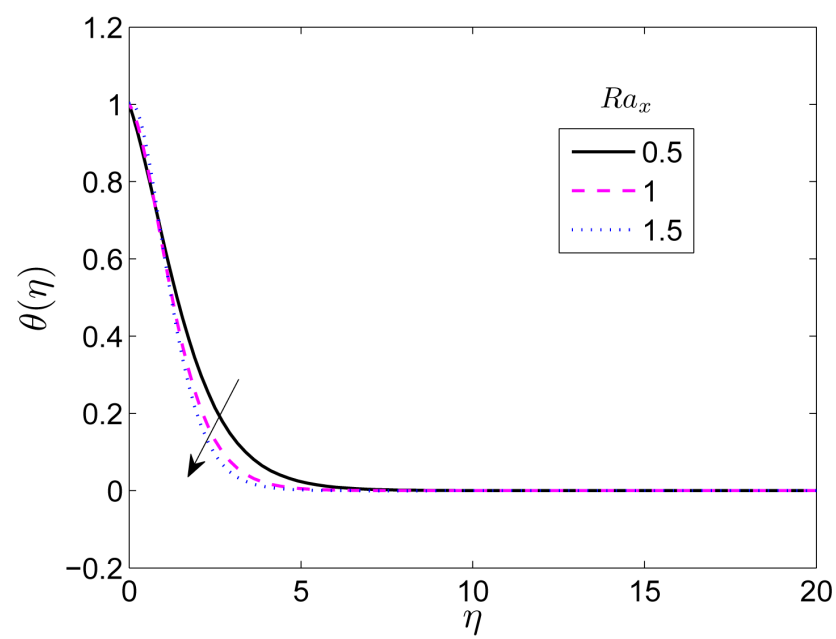

(b)

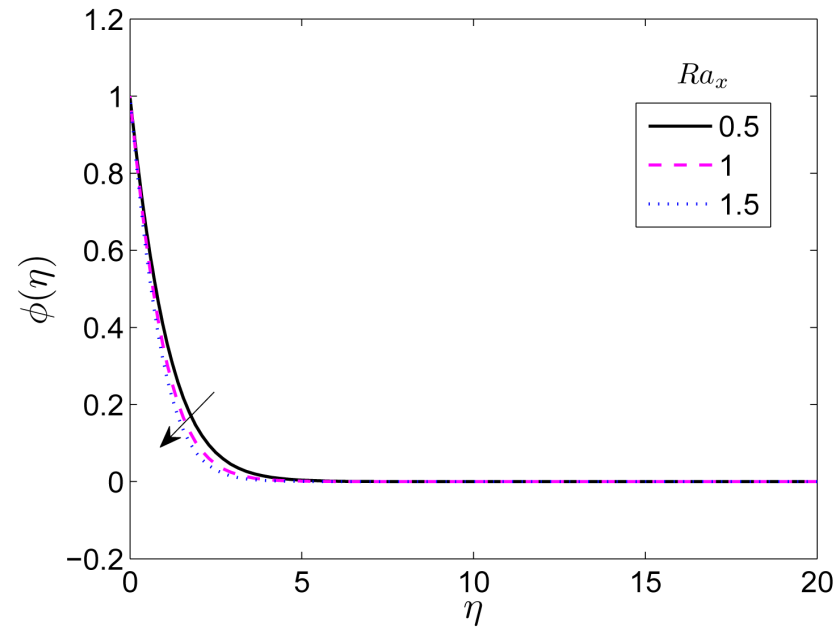

(c)

Figure 1. Effect of thermal rayleigh number $R a_{x}$ on velocity, temperature and concentration profiles. (a) Velocity profile; (b) Temperature profile; (c) Concentration profile. 


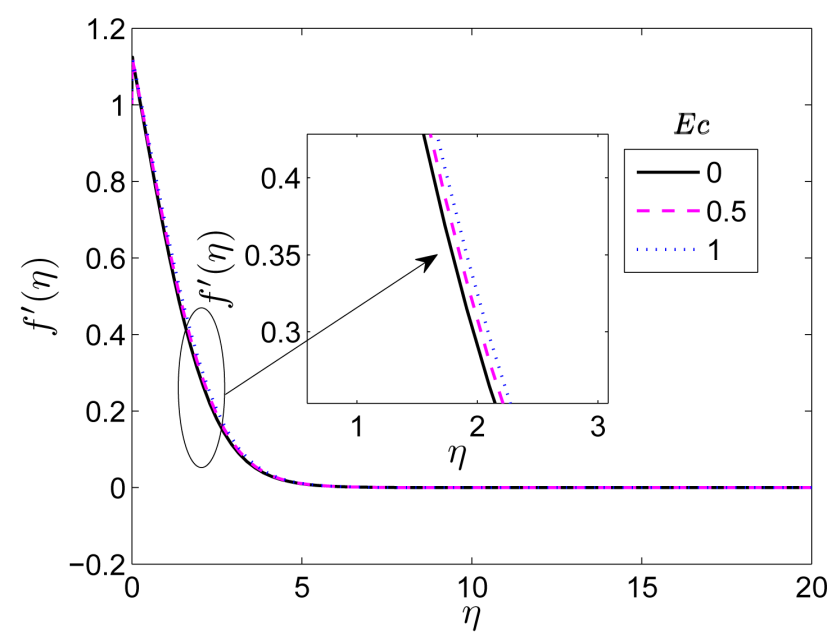

(a)

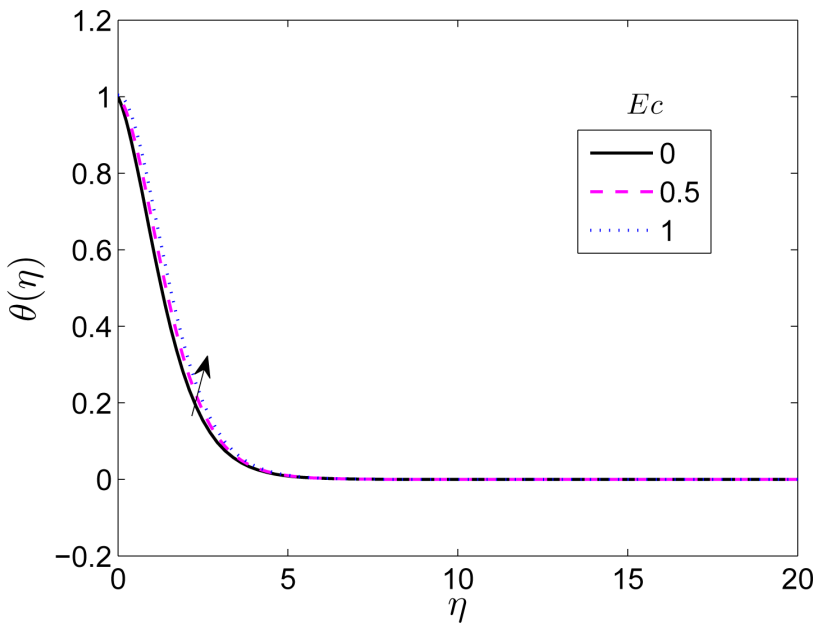

(b)

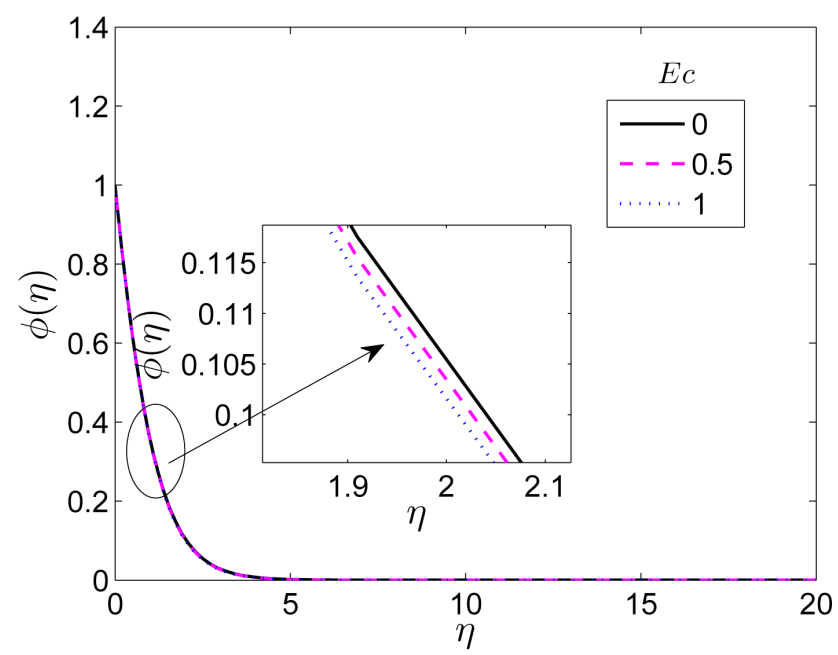

(c)

Figure 2. Effect of Eckert number i.e. viscous dissipation term $E c$ on velocity, temperature and concentration profiles. (a) Velocity profile; (b) Temperature profile; (c) Concentration profile. 


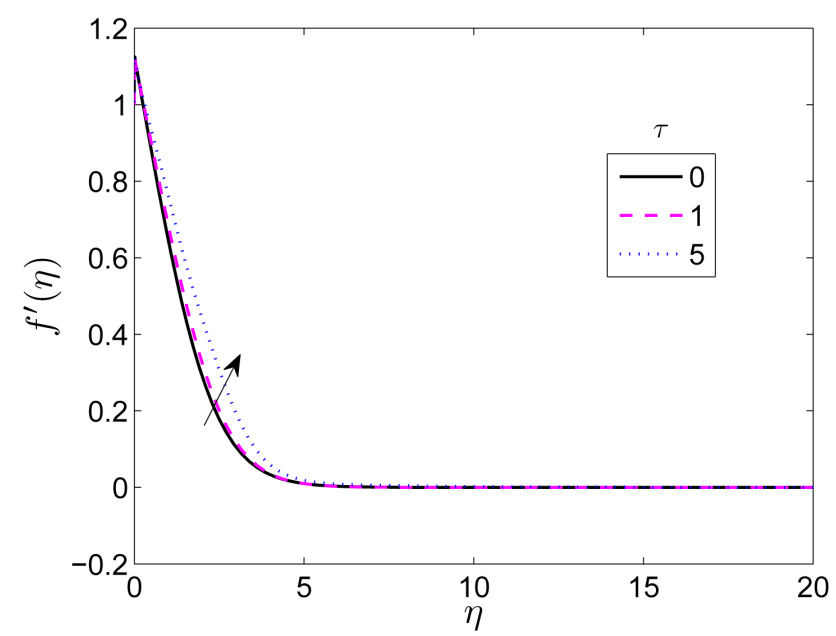

(a)

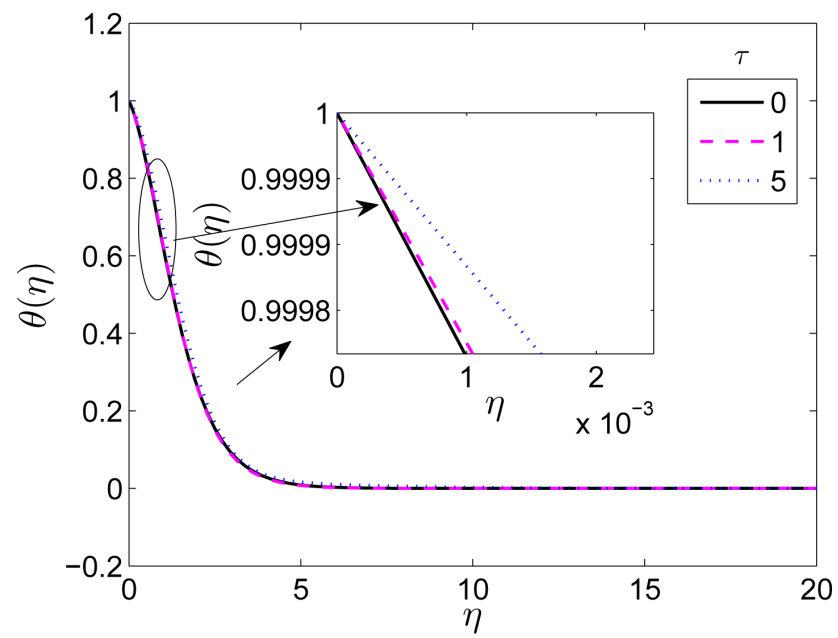

(b)

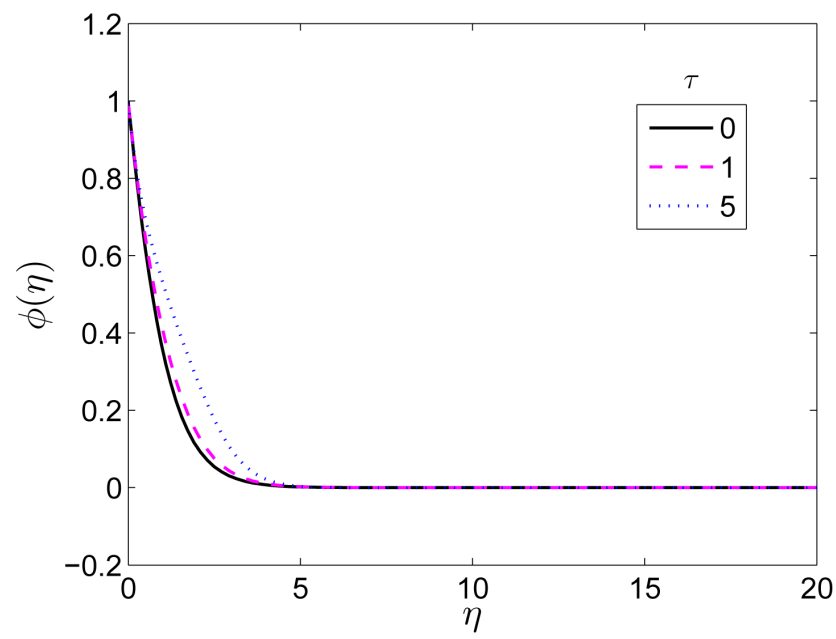

(c)

Figure 3. Effect of thermophoresis parameter $\tau$ on velocity, temperature and concentration profiles. (a) Velocity profile; (b) Temperature profile; (c) Concentration profile. 


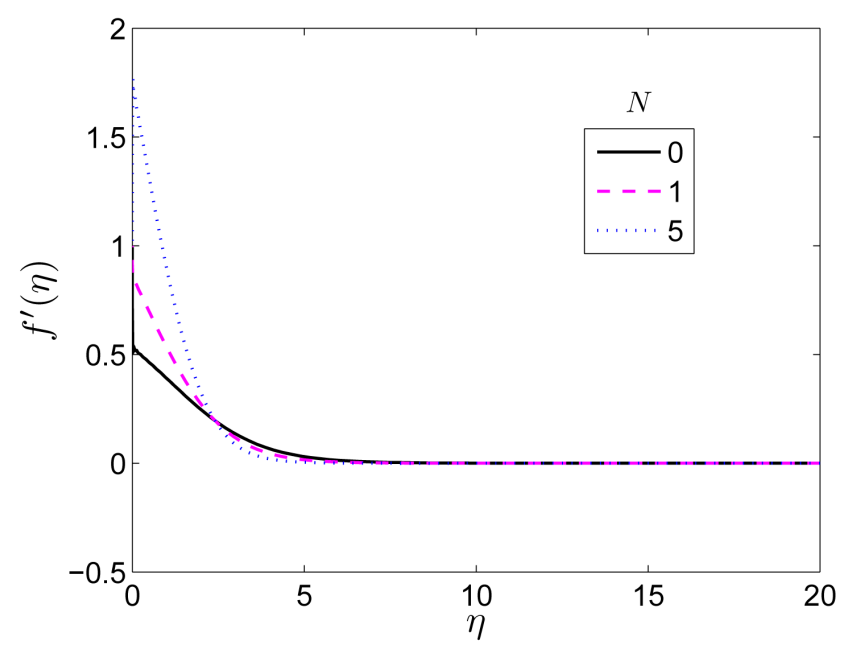

(a)

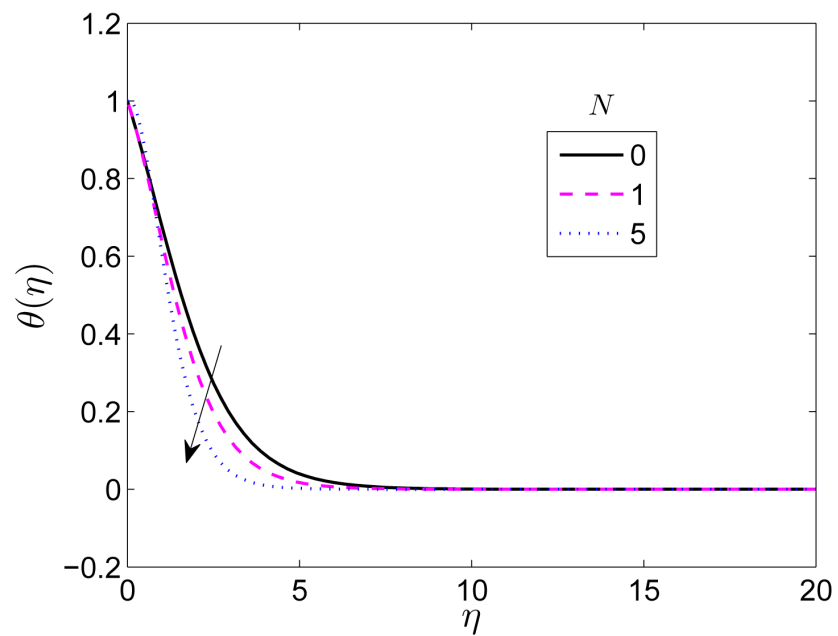

(b)

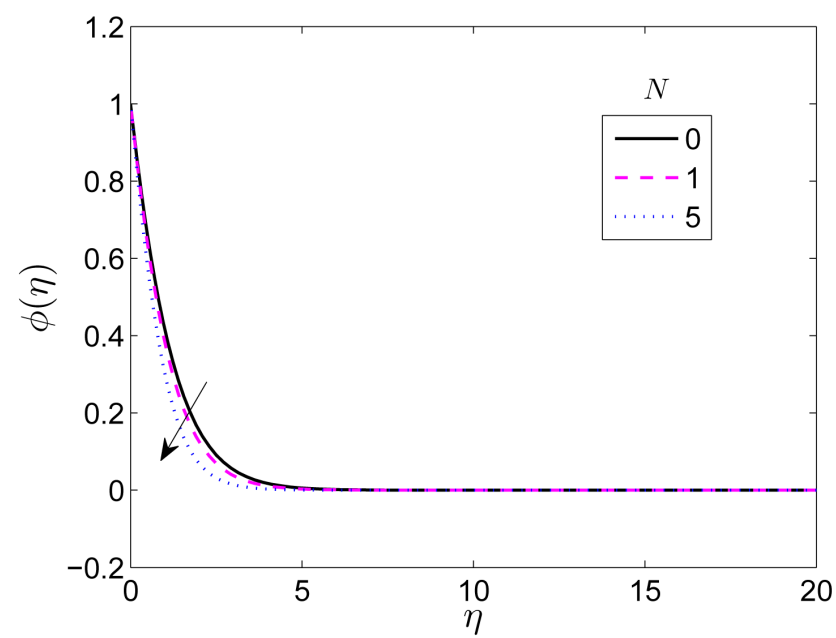

(c)

Figure 4. Effect of bouyancy ratio parameter $N$ on velocity, temperature and concentration profiles. (a) Velocity profile; (b) Temperature profile; (c) Concentration profile. 


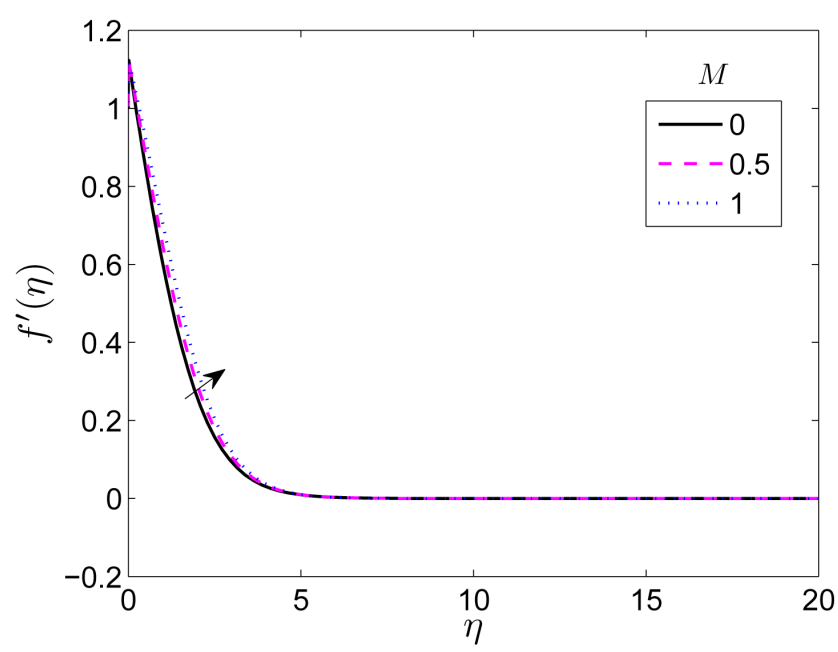

(a)

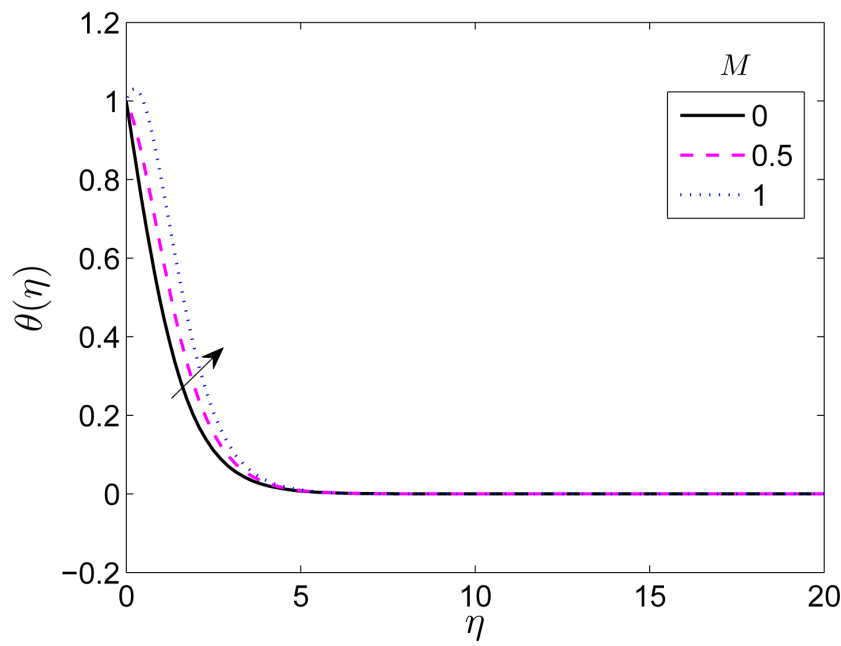

(b)

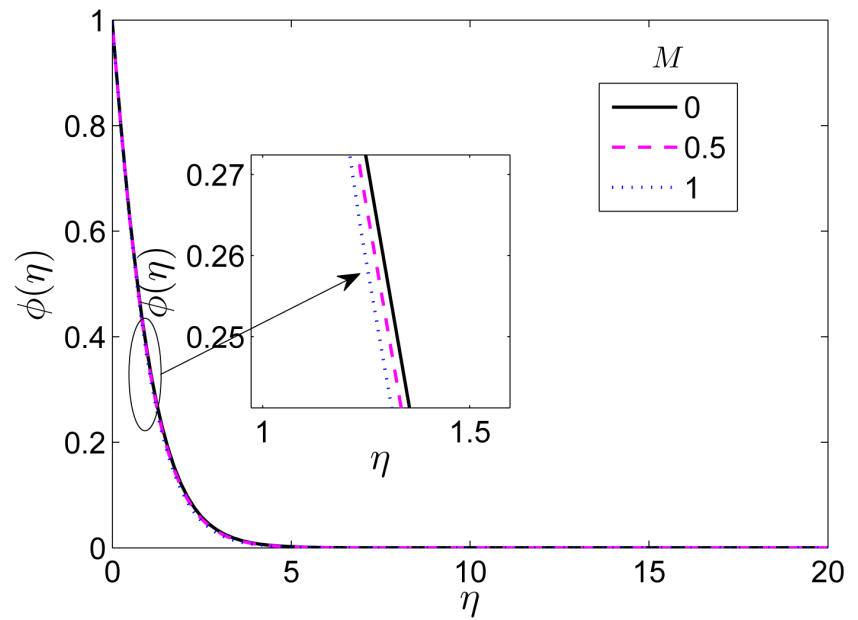

(c)

Figure 5. Effect of magnetic parameter $M$ on velocity, temperature and concentration profiles. (a) Velocity profile; (b) Temperature profile; (c) Concentration profile. 


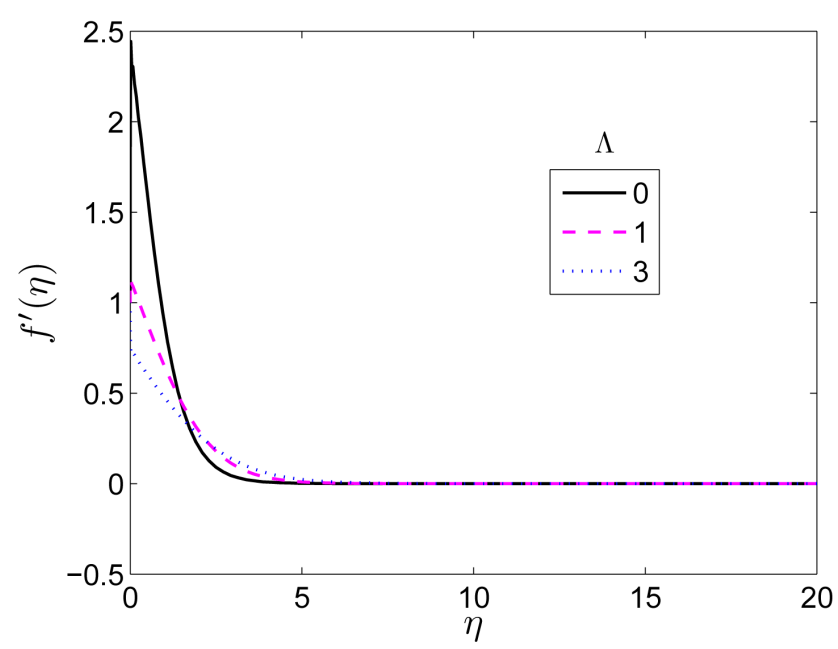

(a)

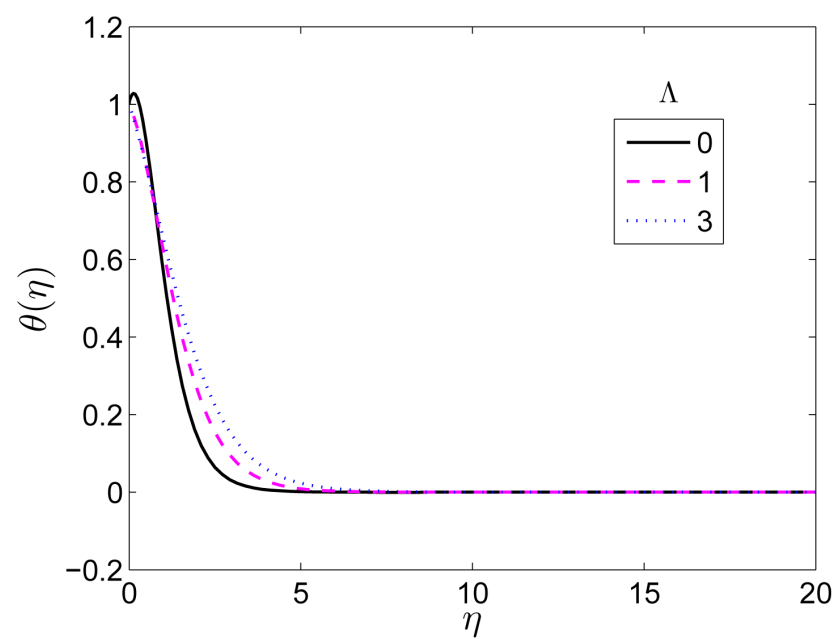

(b)

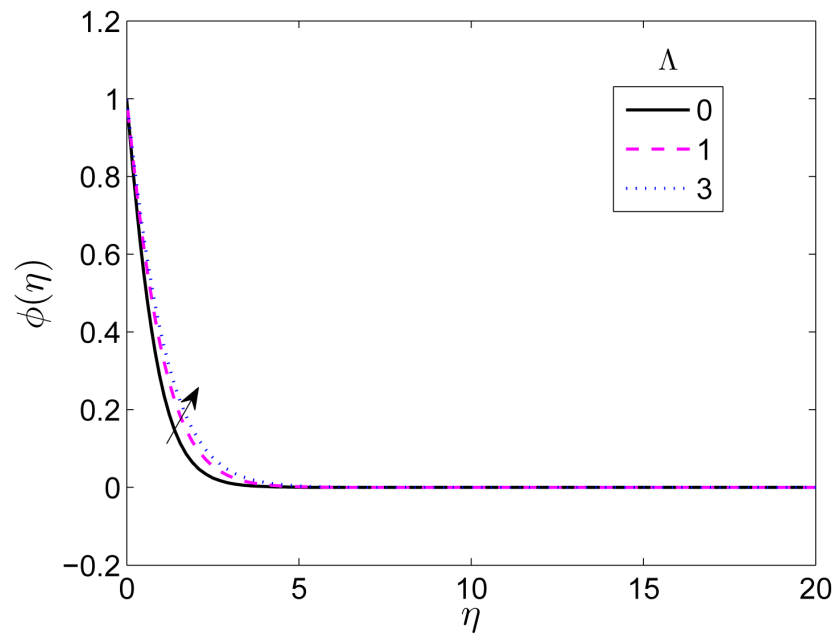

(c)

Figure 6. Effect of inertial parameter $\Lambda$ on velocity, temperature and concentration profiles. (a) Velocity profile; (b) Temperature profile; (c) Concentration profile. 


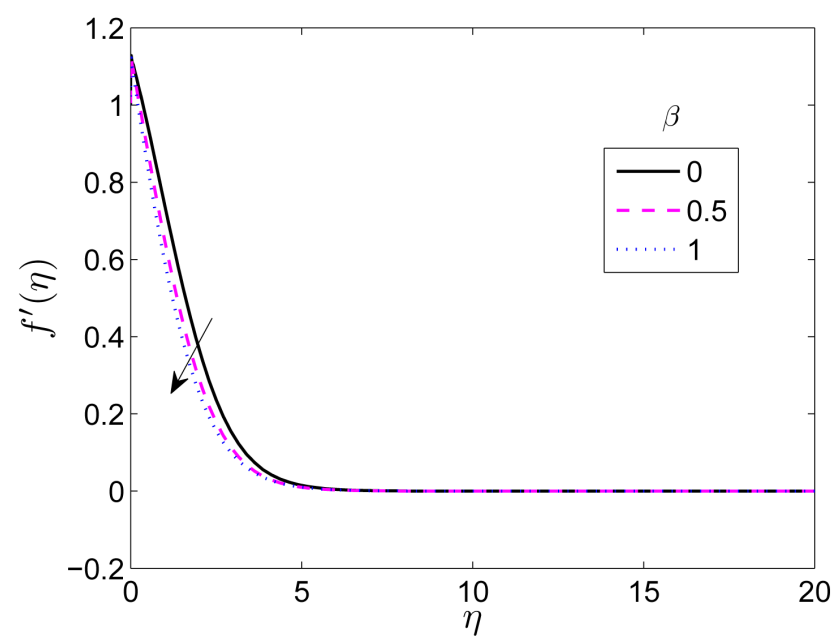

(a)

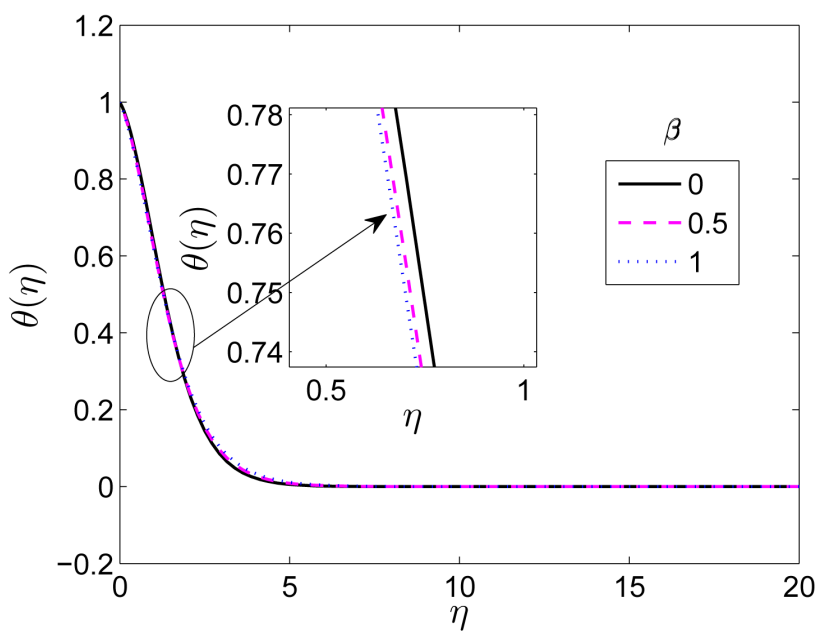

(b)

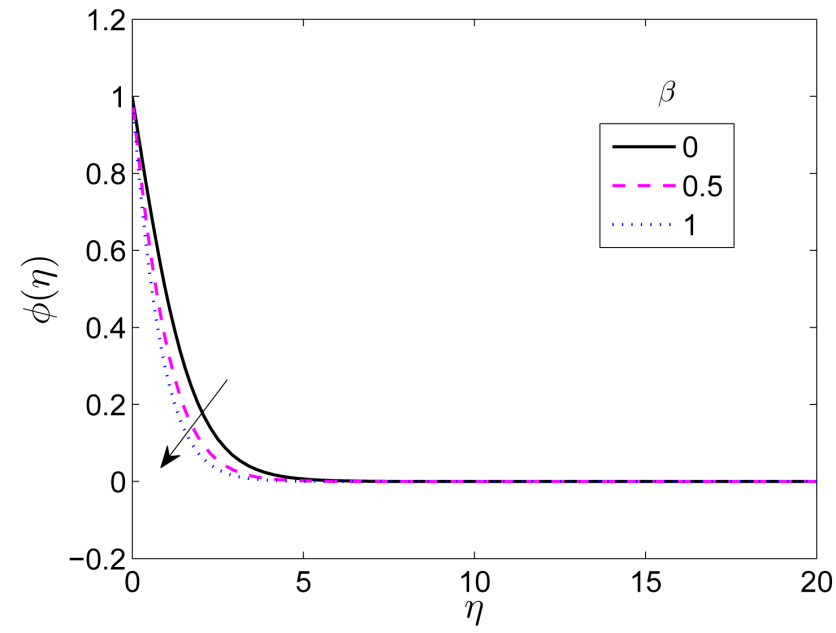

(c)

Figure 7. Effect of chemical reaction parameter $\beta$ on velocity, temperature and concentration profiles. (a) Velocity profile; (b) Temperature profile; (c) Concentration profile. 


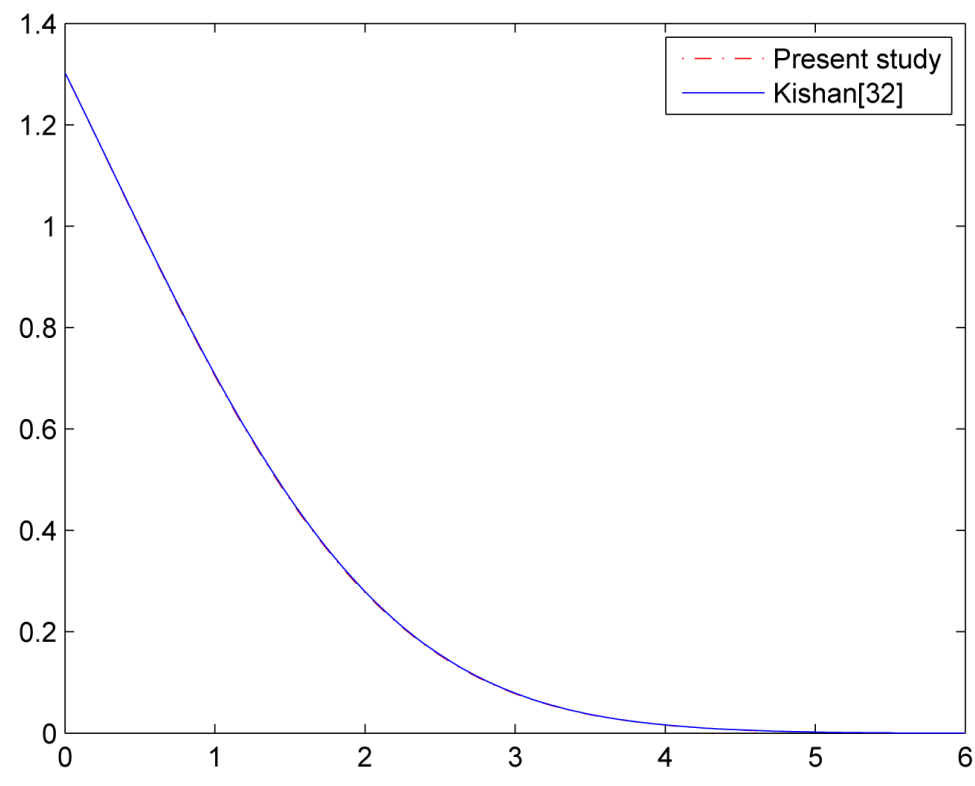

Figure 8. Comparison of velocity profiles of the fluid with the model solved by Kishan [37]. Velocity profile.

parameter $R a_{x}$ on the dimensionless velocity,temperature and concentration profiles of the flow.We note that when $R a_{x} \gg 1$, the flow is dominated by natural convection, while when $R a_{x} \ll 1$, forced convection dominates the flow regime. Hence, when $R a_{x}=1$, the effects of natural convection and forced convection achieve equal importance and flow is dominated by mixed convection conditions. Figure 1(a) shows that velocity of the flow within the boundary layer increases as thermal rayleigh number $R a_{x}$ increase due to the effect of buoyancy force. When the free stream and the boundary force are in opposite direction, the bouyancy force retard the fluid on the boundary layer acting somewhat like an adverse pressure gradient. From Figure 1(b) and Figure 1(c), we observed that the temperature and concentration profile of the flow decrease with increasing value of $R a_{x}$.

Figures 2(a)-(c) illustrate the influence of Eckert number Ec, a viscous dissipation term on the velocity, temperature and concentration distribution. It is observed from these figures that $E c$ have quite opposite effects on the velocity and the associated thermal boundary layer thickness. That is, velocity and temperature profile of the flow increase with increase in the the viscous dissipation term. This further establishes the fact that Eckert number $E c$ enhances temperature distribution in a mixed convective flow. Whereas, concentration profile decreases more quickly for an increasing value of Ec. Figures 3(a)-(c) show the influence of thermophoresis parameter $\tau$ on the dimensionless velocity, dimensionless temperature and concentration in the presence of viscous dissipation. In Figure 3(a), as a result of viscous dissipation effect which acts as source of internal heat to the flow,the velocity profile increase as a result of increase in thermophoresis parameter. This is accompanied by a slight increase in fluid temperature and concentration as seen in Figure 3(b) and Figure 3(c) respectively. Figure 3(c) illustrates the influence of the thermophoretic parameter on the concentration profiles. It is seen that concentration of the fluid increases with an increase of the thermophoretic parameter $\tau$. So, thermophoretic parameter is expected to alter the concentration boundary layer significantly.

Figure 4(a) displays the effects of the ratio of buoyancy parameter $N$ on the velocity, temperature and concentration profile of the fluid. The effects of the ratio of the buoyancy parameter are similar to that of a local buoyancy parameter in a qualitative way. We noticed that the fluid velocity increases more rapidly in the case of increasing buoyancy parameter. It is observed that velocity increases in the presence of aiding flows $(N>0)$. This is because the practical effect of buoyancy ratio $N$ is to enhance the rate of surface heat transfer. Increase in the ratio of the buoyancy parameter correspond to a decrease in the temperature and concentration as observed in Figure 4(b) and Figure 4(c) respectively.

In Figure 5(a), the velocity of the flow increases with increase in magnetic force acting on the the particles of the fluid. This is because the variation of $M$ leads to the variation of Lorentz force due to the magnetic field, and 
the Lorentz force produces more resistance to the transport phenomena. Therefore, the momentum boundary layer thickness becomes larger, and the separation of the boundary layer occurs earlier. In Figure 5(b), the fluid temperature increases with an increase in the Magnetic strength. That is, for increasing $M$ it means that magnetic field actually influenced flow system by increasing the values of temperature in the flow field and then decreasing the gradient at the wall and increases thickness of the thermal boundary layer. While in Figure 5(c), the concentration profile of the flow decreases with increase in the Magnetic parameter M.

The influence of inertia parameter $\Lambda$ is illustrated on the velocity, temperature and concentration profiles of the flow as shown in Figures 6(a)-(c). It is observed that an increase in the value of inertia parameter $\Lambda$ caused a reduction in the maximum velocity attained by the flow. This occurs as a result of increase in the formation of drag within the porous medium when the inertia effect is included. While in Figure 6(b) and Figure 6(c), the temperature and concentration profiles in the boundary layer increase as a result of increase in the inertia parameter and consequently, the thermal and momentum boundary layers become thicker. Figure 7(c) shows the variation of the concentration distribution across the boundary layer for various values of the chemical reaction parameter $\beta$. It is seen that the effect of increasing values of the chemical reaction parameter result to reduction in the velocity, temperature and concentration distribution across the boundary layer. The reason being that chemical reaction increases the rate of interfacial mass transfer. Figure 8 compared the velocity profile of the flow analyzed by [27] and the present study in the absence of magnetic field, chemical reaction and magnetic field. It is observed that the solution obtained by SHAM is in good agreement with Quasilinearization technique used by [27].

The effects of thermophoresis, chemical reaction, Hartmann number, on heat flux and mass flux are presented in Tables 1-3 respectively.

As defined previously in the initial/boundary conditions (14), the velocity, temperature and concentration distributions decay as $\eta$ tends to infinity. The numerical results in Table 1 compared solutions obtained from present model with the one analyzed by [27] on the local heat transfer rate and sherwood number. It is observed that both local heat transfer rate and sherwood number increase as the thermophoresis paramter increases in the flow domain. This further established the accuracy of our results when compared with the earlier work of [27].

\begin{tabular}{|c|c|c|c|c|}
\hline \multirow{2}{*}{$\begin{array}{c}\text { Parameter } \\
\tau\end{array}$} & \multicolumn{2}{|c|}{ Present study } & \multicolumn{2}{|c|}{ N. Kishan [27] when $H a=0$} \\
\hline & $-\theta^{\prime}(0)$ & $-\phi^{\prime}(0)$ & $-\theta^{\prime}(0)$ & $-\phi^{\prime}(0)$ \\
\hline 0.0 & 0.44698 & 0.40591 & 0.44632 & 0.40305 \\
\hline 0.05 & 0.44996 & 0.41663 & 0.44578 & 0.41264 \\
\hline 0.1 & 0.44583 & 0.42201 & 0.44515 & 0.42234 \\
\hline 0.5 & 0.44308 & 0.49971 & 0.44062 & 0.50022 \\
\hline 1.0 & 0.43552 & 0.5906 & 0.43525 & 0.59970 \\
\hline
\end{tabular}

Table 2. SHAM solution for different values of chemical reaction parameter $\beta$ when

$\operatorname{Pr}=0.73, E c=0.01, R a_{x}=0.8, \Lambda=1, S_{c}=0.62, N=1, M=0.5, \tau=0.5, \hbar_{f}=\hbar_{\theta}=\hbar_{\phi}=-0.5$.

\begin{tabular}{ccc}
\hline$\beta$ & $-\theta^{\prime}(0)$ & $-\phi^{\prime}(0)$ \\
\hline 0 & 0.43842 & 0.49973 \\
0.5 & 0.41945 & 0.88663 \\
1 & 0.41004 & 1.14647 \\
1.5 & 0.40398 & 1.35534 \\
2.0 & 0.39954 & 1.53512 \\
\hline
\end{tabular}


Table 3. SHAM solution for different values of magnetic parameter $M$ when $\operatorname{Pr}=0.73, E c=0.01, R a_{x}=0.8, \Lambda=1, S_{c}=0.62, N=1, \tau=0.5, \beta=0.5, \hbar_{f}=\hbar_{\theta}=\hbar_{\phi}=-0.5$.

\begin{tabular}{ccc}
\hline$M$ & $-\theta^{\prime}(0)$ & $-\phi^{\prime}(0)$ \\
\hline 0 & 0.39951 & 1.53512 \\
0.2 & 0.42056 & 0.88687 \\
0.4 & 0.41988 & 0.88673 \\
0.6 & 0.41905 & 0.88655 \\
0.8 & 0.41834 & 0.88632 \\
1.0 & 0.41755 & 0.88611 \\
\hline
\end{tabular}

Table 2 illustrates the influence of chemical reaction parameter $\beta$ on the flow field. It can be seen clearly that local heat transfer rate reduces for every increment in the chemical reaction parameter whereas sherwood number increases with increase in the chemical reaction parametr. Obviously, thermophoresis has direct impact on the concentration profile rather than on the flow velocity and temperature distributions as shown in Table 2. As the chemical reaction species in the flow are multiplied, higher fluid composition can be flushed away from the surface as given in Table 2. Based on Table 3, it can be concluded that as the Hartmann number increases, the Lorentz force due to electromagnetism increases ,leading to increment in internal heat transfer rate.

\section{Conclusions}

In this paper, we have used the spectral homotopy analysis method (SHAM) to solve a second-order nonlinear boundary value problem that governs the two-dimensional steady darcy-forcheimer mixed convection flow in fluid saturated porous media in the presence of chemical reaction and viscous dissipation. The non-linear momentum, energy and species boundary layer equations are transformed into ordinary differential equations using suitable similarity variables.The transformed boundary layer equations are solved using SHAM. We have discussed the effects of magnetic field,chemical reaction,viscous dissipation and thermophoresis on the flow profiles. The main observations are as follows:

- Due to stronger magnetic field the dimensioless velocity decreases whereas, temperature and concentration distributions increase.

- An increase in the ratio of the buoyancy parameter $N$ corresponds to an increase in velocity profile $f^{\prime}(\eta)$ but lead to decrease in the temperature $\theta(\eta)$ and concentration $\phi(\eta)$ distributions.

- The fluid velocity and temperature rise with an increase in Eckert number $E c$, a viscous dissipation term but caused a reduction the concentration profile.

- The influence of thermophoresis parameter on the local heat transfer rate is very significant when compared with the sherwood number.

- Using magnetic field we can control the heat and mass transfer flow characteristics.

- The species concentration profiles increases significantly for the increasing chemical reaction parameter $\beta$ and but opposite trend is observed by the influence of thermophoretic parameter $\tau$ on the local heat transfer.

\section{Acknowledgements}

We thank the editor and the referee for their useful comments.

\section{References}

[1] Nield, D.A. (1968) Onset of Thermohaline Convection in a Porous Medium. Water Resources Research, 4, 553-560. http://dx.doi.org/10.1029/WR004i003p00553

[2] Bejan, A. and Khair, K.R. (1985) Heat and Mass Transfer by Natural Convection in a Porous Medium. International Journal of Heat and Mass Transfer, 28, 909-918. http://dx.doi.org/10.1016/0017-9310(85)90272-8

[3] Yucel, A. (1990) Natural Convection Heat and Mass Transfer along a Vertical Cylinder in a Porous Medium. International Journal of Heat and Mass Transfer, 33, 2265-2274. http://dx.doi.org/10.1016/0017-9310(90)90125-E 
[4] Lai, F.C. and Kulaki, F.A. (1991) Oscillatory Mixed Convection in Horizontal Porous Layers Locally Heated from Below. International Journal of Heat and Mass Transfer, 34, 887-890. http://dx.doi.org/10.1016/0017-9310(91)90134-Z

[5] Rami, Y.J., Fawzi, A. and Abu-Al-Rub, F. (2001) Darcy-Forchheimer Mixed Convection Heat and Mass Transfer in Fluid Saturated Porous Media. International Journal of Numerical Methods for Heat \& Fluid Flow, 11, 600-618. http://dx.doi.org/10.1108/09615530110399503

[6] Kremer, D.M., Davis, R.W., Moore, E.F., Maslar, J.E., Burgess Jr., D.R. and Ehrman, S.H. (2003) An Investigation of Particle Dynamics in a Rotating Disk Chemical Vapor Deposition Reactor. Journal of the Electrochemical Society, 150, G127-G139. http://dx.doi.org/10.1149/1.1536180

[7] Hinds, W.C. (1982) Aerosol Technology: Properties, Behavior, and Measurement of Airborne Particles. John Wiley and Sons, New York.

[8] Goldsmith, P. and May, F.G. (1966) Diffusiophoresis and Thermophoresis in Water Vapour Systems. In: Davies, C.N., Ed., Aerosol Science, Academic Press, London, 163-194.

[9] Goren, S.L. (1977) Thermophoresis of Aerosol Particles in Laminar Boundary Layer on Flat Plate. Journal of Colloid and Interface Science, 61, 77-85. http://dx.doi.org/10.1016/0021-9797(77)90416-7

[10] Chang, Y.P., Tsai, R. and Sui, F.M. (1999) The Effect of Thermophoresis on Particle Deposition from a Mixed Convection Flow onto a Vertical Flat Plate. Journal of Aerosol Science, 30, 1363-1378. http://dx.doi.org/10.1016/S0021-8502(99)00023-3

[11] Jayaraj, S., Dinesh, K.K. and Pallai, K.L. (1999) Thermophoresis in Natural Convection with Variable Properties. Heat and Mass Transfer, 34, 469-475. http://dx.doi.org/10.1007/s002310050284

[12] Selim, A., Hossain, M.A. and Rees, D.A.S. (2003) The Effect of Surface Mass Transfer on Mixed Convection Flow Past a Heated Vertical Flat Permeable Plate with Thermophoresis. International Journal of Thermal Sciences, 42, 973982. http://dx.doi.org/10.1016/S1290-0729(03)00075-9

[13] Chamkha, A.J. and Pop, I. (2004) Effects of Thermophoresis Particle Deposition in Free Convection Boundary Layer from a Vertical Flat Plate Embedded in a Porous Medium. International Communications in Heat and Mass Transfer, 31, 421-430. http://dx.doi.org/10.1016/j.icheatmasstransfer.2004.02.012

[14] Murti, A.S.N., Kameswaran, P.K., Poorna Kantha, T. and Acharyulu, A.A.V.L.A.S. (2012) Numerical Study on MHD Mixed Convection Flow with Dispersion and Chemical Reaction over a Vertical Plate in Non-Darcy Porous Medium. International Journal of Mathematical Archive, 3, 534-549.

[15] Muhaimin, I., Kandasamy, R., Hashim, I. and Ruhaila, K. (2008) Thermphoresis and Chemical Reaction Effects on Non-Darcy MHD Mixed Convective Heat and Mass Transfer past a Porous Wedge in the Presence of Suction or Injection. Selcuk Journal of Applied Mathematics, 9, 77-95.

[16] Salem, A.M. (2006) Coupled Heat and Mass Transfer in Darcy-Forchheimer Mixed Convection from a Vertical Flat Plate Embedded in a Fluid-Saturated Porous Medium under the Effects of Radiation and Viscous Dissipation. Journal of the Korean Physical Society, 48, 409-413.

[17] Srinivasacharya, D. and Swamy Reddy, G. (2012) Chemical Reaction and Radiation Effects on Natural Convective in Porous Medium Saturated with Power-Law Fluid. Frontiers in Heat and Mass Transfer, 3, 1-9.

[18] Huang, J.S., Tsai, R.Y. and Huang, K.H. (2012) Numerical Study of Thermophoresis on Aerosol Particle Deposition from Hiemenz Flow through Porous Medium onto a Stretching Surface. Journal of Marine Science and Technology, 20, 163-172.

[19] Postelnicu, A. (2004) Influence of a Magnetic Field on Heat and Mass Transfer by Natural Convection from Vertical Surfaces in Porous Media Considering Soret and Dufor Effects. International Journal of Heat and Mass Transfer, 47, 1467-1472. http://dx.doi.org/10.1016/j.ijheatmasstransfer.2003.09.017

[20] Ahammad, M.U., Obayedullah, Md. and Rahman, M.M. (2013) Analysis of MHD Free Convection Flow along a Vertical Porous Plate Embedded in a Porous Medium with Magnetic Field and Heat Generation. Journal of Engineering e-Transaction, 8, 10-18.

[21] Sonth, R.M., Khan, S.K., Abel, M.S. and Prasad, K.V. (2002) Heat and Mass Transfer in a Visco-Elastic Fluid over an Accelerating Surface with Heat Source/Sink and Viscous Dissipation. Heat and Mass Transfer, 38, 213-220. http://dx.doi.org/10.1007/s002310100271

[22] Venkateswarlu, M., Ramana Reddy, G.V. and Lakshimi, D.V. (2013) Effects of Chemical Reaction and Heat Generation on MHD Boundary Layer Flow of a Moving Vertical Plate with Suction and Dissipation. Engineering International, 1, 27-38.

[23] Muhaimin, I., Kandasamy, R., Khamis, A.B. and Roslan, R. (2013) Effect of Thermophoresis Particle Deposition and Chemical Reaction on Unsteady MHD Mixed Convective Flow over a Porous Wedge in the Presence of Temperature Dependent Viscosity. Nuclear Engineering and Design, 261, 95-106. 
http://dx.doi.org/10.1016/j.nucengdes.2013.03.015

[24] Mahdy, A. (2013) Thermophoresis Particle Deposition and Variable Viscosity Effects on Non-Darcy Free Convection in a Fluid Saturated Porous Media with Uniform Suction/Injection. Latin American Applied Research, 43, 113-119.

[25] Alam, M.S. and Rahman, M.M. (2013) On the Effectiveness of Variable Heat and Mass Fluxes on Hydromagnetic Free Convection and Mass Transfer Flow along an Inclined Permeable Stretching Surface with Thermophoresis. International Journal of Energy and Technology, 5, 1-10.

[26] RamReddy, Ch., Murthy, P.V.S.N., Chamkha, A.J. and Rashad, A.M. (2013) Influence of Viscous Dissipation on Free Convection in a Non-Darcy Porous Medium Saturated Medium with Nanofluid in the Presence of Magnetic Field. The Open Transport Phenomena Journal, 5, 20-29.

[27] Kishan, N. and Maripala, S. (2012) Thermophpresis and Viscous Dissipation Effects on Darcy-Forcheimer MHD Mixed Convection in a Fluid Saturated Porous Media. Advances in Applied Science Research, 3, 60-74.

[28] Shrivastava, U.N. and Usha, S. (1987) Effect of Magnetic Field on Boundary Layer Thickness and Skin Friction at the Surface. Indian Journal of Pure and Applied Mathematics, 18, 741-751.

[29] Noghrehabadi, A., Ghalambaz, M., Izadpanahi, E. and Pourrajab, R. (2014) Effect of Magnetic Filed on the Boundary Layer Flow, Heat and Mass Transfer of Nanofluids over s Stretching Cylinder. Journal of Heat and Mass Transfer Research, 1, 9-16.

[30] Ishak, A., et al. (2008) Effect of a Uniform Transverse Magnetic Field on the Stagnation Point Flow over a Stretching Vertical Sheet. Journal of Heat and Mass Transfer, 44, 921-927.

[31] Isa, S.S.P.M., Arifin, N.M., Nazar, R., Bachok, N., Ali, F.M. and Pop, I. (2014) Effect of Magnetic Field on Mixed Convection Boundary Layer Flow over an Exponentially Shrinking Vertical Sheet with Suction. International Journal of Mechanical, Aerospace, Industrial and Mechatronics Engineering, 8, 1519.

[32] Talbot, L., Cheng, R.K., Scheffer, R.W. and Wills, D.R. (1980) Thermophoresis of Particles in a Heated Boundary Layer. Journal of Fluid Mechanics, 101, 737-758. http://dx.doi.org/10.1017/S0022112080001905

[33] Batchelor, G.K. and Shen, C. (1985) Thermophoretic Deposition of Particles in Gas Flowing over Cold Surfaces. Journal of Colloid and Interface Science, 107, 21-37. http://dx.doi.org/10.1016/0021-9797(85)90145-6

[34] Liao, S.J. (2003) Beyond Perturbation: Introduction to the Homotopy Analysis Method. Chapman and Hall/CRC Press, Boca Raton. http://dx.doi.org/10.1201/9780203491164

[35] Liao, S.J. (2012) Homotopy Analysis Method in Nonlinear Differential Equations. Springer and Higher Education Press, Berlin \&Beijing. http://dx.doi.org/10.1007/978-3-642-25132-0

[36] Motsa, S.S., Sibanda, P. and Shateyi, S. (2010) A New Spectral-Homotopy Analysis Method for Solving a Nonlinear Second Order BVP. Communications in Nonlinear Science and Numerical Simulation, 15, 2293-2302. http://dx.doi.org/10.1016/j.cnsns.2009.09.019

[37] Motsa, S.S., Sibanda, P., Awad, F.G. and Shateyi, S. (2010) A New Spectral-Homotopy Analysis Method for the MHD Jeffery-Hamel Problem. Computers \& Fluids, 39, 1219-1225.

[38] Canuto, C., Hussaini, M.Y., Quarteroni, A. and Zang, T.A. (1988) Spectral Methods in Fluid Dynamics. SpringerVerlag, Berlin. http://dx.doi.org/10.1007/978-3-642-84108-8

[39] Trefethen, L.N. (2000) Spectral Methods in MATLAB. SIAM, Philadelphia. http://dx.doi.org/10.1137/1.9780898719598 


\section{Nomenclature}

\section{English Symbols}

\begin{tabular}{|c|c|}
\hline$U_{w}(x)$ & stretching velocity of the plate \\
\hline$T_{\infty}$ & free stream temperature of the surrounding fluid \\
\hline$u, v$ & velocity components in $\mathrm{x}$ - and $\mathrm{y}$-respectively \\
\hline$g$ & gravitational force due to acceleration \\
\hline$D_{m}$ & coefficient of mass diffusivity \\
\hline$C_{p}$ & the specific heat at constant pressure \\
\hline$T_{m}$ & reference temperature \\
\hline$K_{1}$ & Darcy permeability \\
\hline$k^{*}$ & thermophoretic coefficient \\
\hline$M$ & Magnetic parameter \\
\hline$R a_{x}$ & thermal Rayleigh number \\
\hline$N$ & buoyancy ratio \\
\hline$P_{r}$ & Prandtl number \\
\hline$S_{c}$ & Schmidtl number \\
\hline$C_{1}$ & Cunningham correction factor \\
\hline Kn & Knudsen number \\
\hline$C_{m}$ & real constant \\
\hline$C_{\mathrm{s}}$ & real constant \\
\hline$C_{t}$ & real constant \\
\hline$C_{f}$ & skin-friction coefficient \\
\hline$f$ & dimensionless stream function \\
\hline $\mathrm{Nu}$ & Nusselt number \\
\hline Sh & Sherwood number \\
\hline$f_{w}$ & suction/injection parameter \\
\hline$C_{\infty}$ & free stream concentration \\
\hline$T$ & fluid temperature \\
\hline C & fluid concentration \\
\hline \multicolumn{2}{|c|}{ Greek Symbols } \\
\hline$\mu$ & dynamic viscosity \\
\hline$\rho$ & density \\
\hline$\beta t$ & volumetric coefficient of thermal expansion \\
\hline$\beta c$ & volumetric coefficient of expansion \\
\hline$\alpha$ & thermal diffusivity \\
\hline$\beta_{o}$ & magnetic field of constant strength \\
\hline
\end{tabular}


A. I. Fagbade et al.

\section{Continued}

$\sigma^{*}$

v

$\lambda_{g}$

$\lambda_{p}$

$\phi$

$\eta$

$\psi$

$\beta$

$\Lambda$

$\tau$ electrical conductivity

kinematic viscosity

thermal conductivity of the fluid

thermal conductivity of the diffused particle

dimensionless concentration

similarity variable

stream function

dimensionless temperature

chemical reaction parameter

inertial parameter

thermophoretic parameter 